\title{
ON COVERINGS AND HYPERALGEBRAS OF AFFINE ALGEBRAIC GROUPS
}

\author{
BY
}

\author{
MITSUHIRO TAKEUCHI
}

\begin{abstract}
Over an algebraically closed field of characteristic zero, the universal group covering of a connected affine algebraic group, if such exists, can be constructed canonically from its Lie algebra only. In particular the isomorphism classes of simply connected affine algebraic groups are in 1-1 correspondence with the isomorphism classes of finite dimensional Lie algebras of some sort. We shall consider the counterpart of these results (due to Hochschild) in case of a positive characteristic, replacing the Lie algebra by the "hyperalgebra". We show that the universal group covering of a connected affine algebraic group scheme can be constructed canonically from its hyperalgebra only and hence, in particular, that the category of simply connected affine algebraic group schemes is equivalent to a subcategory of the category of hyperalgebras of finite type which contains all the semisimple hyperalgebras.
\end{abstract}

Introduction. Let $k$ be an arbitrary field of arbitrary characteristic. Let $\mathbb{B}$ and $\mathscr{F}$ be connected affine algebraic $k$-group schemes. If $\eta: \mathscr{F} \rightarrow \mathbb{J}$ is an epimorphism of $k$-group schemes whose kernel $\operatorname{Rex}(\eta$ ) (in the category of $k$ group schemes) is a finite etale $k$-group scheme, then the pair $(\mathscr{l}, \eta)$ is called an etale group covering of $\mathbb{B}$. The $k$-group scheme $\mathbb{B}$ is simply connected (or (SC)), if it has no nontrivial etale group covering. An etale group covering $\gamma: \mathbb{B}^{*} \rightarrow \mathbb{B}$ is called a universal group covering of $\mathbb{B}$, if the $k$-group scheme $\mathbb{B}^{*}$ is (SC). Such a universal group covering $\left(\mathbb{S}^{*}, \gamma\right)$, if it exists, should satisfy the following universal mapping property and hence will be determined uniquely up to a unique isomorphism.

For each etale group covering $(\mathfrak{L}, \eta)$ of $\mathbb{B}$, there exists a unique homomorphism of $k$-group schemes $\eta^{*}: \mathbb{8}^{*} \rightarrow$ Sृ with $\eta \circ \eta^{*}=\gamma$.

The purpose of this article is to generalize the following result of Hochschild to the case of arbitrary perfect ground field of arbitrary characteristic:

Received by the editors March 22, 1974.

AMS (MOS) subject classifications (1970). Primary 14L15.

Key words and phrases. Group scheme, Hopf algebra, hyperalgebra, group covering, simply connected. 
Theorem (Hochschild [2]). If $k$ is an algebraically closed field of characteristic zero, the following statements hold.

(a) A connected affine algebraic $k$-group scheme \& bas a universal group covering if and only if the radical of $\mathbb{B}$ is unipotent.

(b) Then the universal group covering \& $^{*}$ of $\&$ can be constructed canonically from its Lie algebra $L=\mathrm{Lie}(\mathbb{S})$ only.

(c) In particular, the isomorphism classes of (SC) k-group schemes are in 1-1 correspondence with the isomorphism classes of finite dimensional Lie algebras $L$ over $k$ whose radical $A$ is nilpotent.

More precisely, let $L$ be a finite dimensional Lie algebra over $k$. The universal enveloping algebra $U(L)$ of $L$ has a unique Hopf algebra structure having $L$ as primitive elements. Its dual Hopf algebra $H(L)=U(L)^{0}$ (see \$0. 1 for definition) is a commutative (but not always finitely generated). domain as an algebra. Let $A$ be the radical of $L$ and denote by $\langle A\rangle$ the ideal of $U(L)$ generated by $A$. Those elements of $H(L)$ which annihilate some power of $\langle A\rangle$ form a finitely generated sub-Hopf algebra of $H(L)$ denoted by $B(L)$. Let $B(L)=\operatorname{Spec}(B(L))$ denote the corresponding affine algebraic $k$-group scheme. Suppose that the radical $A$ is nilpotent. Then the $k$-group scheme $B(L)$ is (SC) and its Lie algebra Lie $(B(L))$ is canonically isomorphic with $L$. If in particular $L=[L, L]$, then the radical $A$ is automatically nilpotent and we have $B(L)=H(L)$. Now let $B$ be a connected affine algebraic $k$-group scheme whose radical is unipotent. Let $\Theta(\mathbb{B})$ denote its affine Hopf algebra. The radical $A$ of the Lie algebra $L=\mathrm{Lie}(\$)$ is then nilpotent and the image of the canonical injective homomorphism $\Theta(\Im) \hookrightarrow U(L)^{0}=H(L)$ is contained in $B(L)$. Therefore there results a canonical epimorphism of $k$-group schemes $\gamma: B(L) \rightarrow B$ which proves to be a universal group covering of $B$. In particular every (SC) $k$-group scheme is of the form $B(L)$ with a uniquely determined finite dimensional Lie algebra $L$ whose radical $A$ is nilpotent.

When the characteristic of the field is positive, the byperalgebra plays the same role as the Lie algebra does in case of characteristic zero. The hyperalgebra hy(B) of an affine algebraic $k$-group scheme $\mathbb{B}$ is by definition the irreducible component containing 1 of the dual Hopf algebra $\theta(8){ }^{0}$ of the affine Hopf algebra $\Theta(\mathbb{B})$. Takeuchi $\left[\mathrm{T}_{\mathrm{I}}\right],\left[\mathrm{T}_{\mathrm{II}}\right]$ develops the theory of hyperalgebras of algebraic groups which is completely analogous to the classical theory of Lie algebras of algebraic groups over a field of characteristic zero. The theory of hyperalgebras is briefly summarized in $\$ 0.3$ for convenience of the reader. We have been able to characterize the (SC) $k$-group schemes by their hyperalgebra as follows: 
Theorem. Suppose the base field $k$ is perfect with a positive characteristic p. For each connected affine algebraic (not necessarily smooth) k-group scheme $\mathbb{B}$, the following conditions are equivalent to each other:

(i) \& is (SC).

(ii) The affine Hopf algsbra $\Theta(\mathbb{B})$ is canonically isomorphic with the dual Hopf algebra hy(B) ${ }^{0}$ of the hyperalgebra hy(\&).

(iii) For each locally algebraic (not necessarily affine) $k$-group scheme S, the map

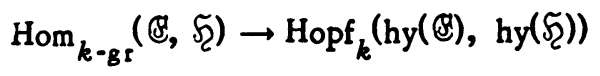

which sends each $f \in \operatorname{Hom}_{k-\mathrm{g} g}\left(\&, \mathcal{F}_{)}\right)$to the induced homomorphism of hyper-

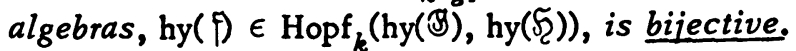

From this theorem, it follows that if a connected affine algebraic $k$-group scheme $(8)$ has a universal group covering $\left(\mathbb{B}^{*}, \gamma\right)$, then the affine Hopf algebra $O\left(\mathbb{B}^{*}\right)$ is canonically isomorphic with the dual Hopf algebra hy(B) ${ }^{0}$ of hy(\&), because hy $(\gamma):$ hy $\left(\mathbb{B}^{*}\right) \simeq$ hy(\&) and $\Theta\left(B^{*}\right) \simeq$ hy $\left(\mathbb{B}^{*}\right)^{0}$. This means that the dual Hopf algebra hy(\&) ${ }^{0}$ is finitely generated, the corresponding $k$ group scheme Spec(hy(B) $)^{0}$ ) is (SC), the hyperalgebra of Spec(hy(B) $\left.{ }^{0}\right)$ is canonically isomorphic with hy(\&) and that the canonical homomorphism of $k$-group schemes Spec $\left(\right.$ hy $\left.(\mathbb{B})^{0}\right) \rightarrow \mathbb{B}$ is a universal group covering of $\mathbb{O}$. Thus the universal group covering of $\mathbb{B}$, if it exists, can be canonically constructed from its byperalgebra hy(B) only. On the other hand, we have

Theorem. Suppose $k$ is perfect and $p>0$. If $\&$ is an (SC) affine alge. braic $k$-group scheme, then $\& / \&, \&]$ is a finite $k$-group scheme, that is its affine Hopf algebra is finite dimensional. If in particular $B$ is smooth and $(S C)$, then $B=[B, B]$ and the radical of $\mathbb{B}$ is unipotent.

Thus our (SC) $k$-group schemes in case of a positive characteristic correspond with the $k$-group schemes $\mathbb{B}(L)$ with $L=[L, L]$ in case of characteristic zero. In particular the 'if' part of the statement (a) of the theorem of Hochschild does not hold in case of a positive characteristic as it stands. (The additive $k$-group scheme $B_{a}$ is (SC) if $p=0$ but not if $p>0$.) But if we replace the condition (SC) by the following concept of simply connectedness relative to $p$, then statement (a) does hold in any characteristic (cf. Miyanishi [4] also).

Let $p^{*}$ denote the characteristic exponent $(=\operatorname{Max}(1, p))$ of $k$. Let $\mathcal{F}_{2}$ and (8) be connected affine algebraic $k$-group schemes. An epimorphism of $k$-group schemes $\eta: \mathscr{F} \rightarrow B$ is called a p-etale group covering of $\mathbb{B}$ if the kernel 
$\operatorname{Rex}(\eta)$ of $\eta$ is a finite etale $k$-group scheme whose order (= the dimension of the affine ring over $k$ ) is relatively prime to $p^{*}$. The $k$-group scheme $\&$ is called $p$-simply connected (or $(\mathrm{SC})_{p}$ ) if it has no nontrivial $p$-etale group covering. By a p-universal group covering of $B$ we mean a $p$-etale group covering (\&*, $\gamma$ ) of $\mathbb{E}$ with $\mathbb{B}^{*}(\mathrm{SC})_{p}$. Such a $p$-universal group covering of $\mathbb{B}$, if it exists, satisfies the same universal mapping property as the usual universal group covering of $\mathbb{B}$ (where of course the p-etale group coverings must take the place of the usual etale group coverings) and hence is uniquely determined up to a unique isomorphism. If $p=0$, being $(\mathrm{SC})_{0}$ is equivalent to being (SC).

Theorem. If $k$ is perfect, a connected smooth affine algebraic $k$-group scheme $\mathbb{B}$ has a p-universal group covering if and only if the radical of $\mathbb{B}$ is unipotent.

In order to obtain the $p$-universal group covering of $\mathbb{B}$ whose radical is unipotent, we must first treat the semisimple $k$-group schemes. Indeed we shall show that every connected semisimple $k$-group scheme has a universal group covering which is at the same time a $p$-universal group covering. Hence if $B_{u}$ denotes the unipotent radical of $B$, the quotient group $B / B$, which is semisimple by assumption, has a $p$-universal group covering $\left(B / \mathbb{S}_{u}\right)^{*}$. If we pull it back along $B \rightarrow \mathbb{B} / \mathbb{B}$, then we obtain a $p$-universal group covering $\mathbb{B}^{*}=\left(B \times \mathrm{C}_{u}(B / B)^{*}\right.$ of $B$.

The case of connected semisimple $k$-group schemes goes as follows. First we consider the case where $k$ is algebraically closed. It is well known that all connected semisimple $k$-group schemes are then described up to isomorphisms by their root system (cf. Satake [5], etc.). Let $\mathbb{B}=\mathbb{B}(X, \nabla)$ denote the connected semisimple $k$-group scheme (i.e., the Chevalley $k$-group scheme) determined by the root system $(X, \nabla)$. Let $X_{0}=\{\nabla\}_{Z}$ be the Z-submodule of $X$ generated by $\nabla$ and $X^{0}$ be the weight module of $(X, \nabla)$, that is

$$
X^{0}=\left\{x \in X_{Q}\left\langle\nabla^{*}, x\right\rangle \subset \mathbf{Z}\right\}
$$

where $\nabla^{*}$ denotes the coroot system of $(X, \nabla)$. We have canonical inclusions $X_{0} \subset X \subset X^{0}$. Traditionally the Chevalley $k-$ group scheme $\&$ is called "simply connected" if $X=X^{0}$ and adjoint if $X=X_{0}$. But the simply connectedness in this sense is not equivalent with our (SC)-ness. That is:

Theorem. Suppose $k$ is algebraically closed. Determine the subgroup $\bar{X}$ of $X^{0}$ containing $X$ by the following condition:

$$
\left(p^{*},[\bar{X}: X]\right)=1 \text { and }\left[X^{0}: \bar{X}\right]=\text { a power of } p^{*} \text {. }
$$


(If $p=0$, then $\bar{X}=X^{0}$.) We have then:

(a) $\mathbb{B}=B(X, \nabla)$ is (SC) if and only if $X=\bar{X}$.

(b) The natural inclusion of reduced root systems $(X, \nabla) \hookrightarrow(\bar{X}, \nabla)$ induces an isogeny of Chevalley k-group schemes $\gamma: \&(\bar{X}, \nabla) \rightarrow \&(X, \nabla)$ (uniquely determined up to inner automorphisms by k-rational points of the maximal torus of $B(\bar{X}, \nabla)$ ) which is a universal group covering, as well as a p-universal group covering, of $\mathbb{B}$.

Thus all "simply connected" Chevalley $k$-group schemes are (SC) in our sense but the converse does not hold. For instance even the adjoint Chevalley $k$-group scheme $\beta G \Omega_{n}=G \mathfrak{F}_{n} /{ }_{n} \mu$ is (SC) when $n$ is a power of $p^{*}$. Miyanishi [4] seems to have missed these circumstances. (Look at the "proof" of [4, Lemma 4].)

Coming back to the case where $k$ is only perfect, if $\&$ is a connected semisimple affine algebraic $k$-group scheme, then $B \otimes \bar{k}$ (where $\bar{k}=$ the algebraic closure of $k$ ), which is semisimple, has the universal group covering $(\otimes \otimes \bar{k})^{*}$. We shall prove that the $\bar{k}$-group scheme $(\otimes \otimes \bar{k})^{*}$ has a "k-form" $\bar{B}$ such that the epimorphism $(\& \otimes \bar{k})^{*} \rightarrow \otimes \otimes \bar{k}$ is "defined over $k$ ". Then $\bar{B}$ is easily seen to be a universal, as well as a $p$-universal, group covering of 8 . In other words the dual Hopf algebra hy(\&) $)^{0}$ is finitely generated and the hyperalgebra of $\bar{B}=\operatorname{Spec}(\text { hy }(B))^{0}$ ), which is "the" universal group covering of $B$, is canonically isomorphic with hy(B). It is clear that the hyperalgebra hy(\&) is smooth and semisimple, in the sense of having no "radical". Conversely we can prove

Theorem. Let $J$ be a smooth semisimple hyperalgebra over $k$ of finite type where $k$ is perfect. Then $J$ is the hyperalgebra of some (SC) semisimple k-group scheme \&) which is uniquely determined, that is $8=\operatorname{Spec}\left(J^{0}\right)$. Thus the category of (SC) semisimple k-group schemes is equivalent to the category of smooth semisimple finite type hyperalgebras over $k$.

Finally we shall conclude this paper by giving an example of (SC) $k$ group schemes which are not reductive.

This article is based on the theories of Hopf algebras, group schemes and hyperalgebras. They are prepared in $\$ 0$.

To avoid confusion, all $k$-group schemes are denoted by German letters, but all Hopf algebras by Latin letters.

$\S 0$. Preliminaries. Throughout the paper $k$ denotes a fixed ground field of characteristic $p$. The characteristic exponent of $k$ is denoted by $p^{*}=$ $\operatorname{Max}(1, p)$. If $V$ is a $k$-vector space, the dual space $\operatorname{Hom}_{k}(V, k)$ is denoted 
by $V^{*}$. A subset $T$ of $V^{*}$ is dense if $T^{\perp}=\left\{v \in V^{*} \mid\langle T, v\rangle=0\right\}=0$. A subspace $W$ of $V$ is cofinite if $V / W$ is finite dimensional.

For each homomorphism of fields $\phi: k \rightarrow K$ and each $k$-vector space $V$, $V \otimes_{\phi} K$ denotes the scalar extension $V \otimes_{k} K$. In particular $V^{(p)}$ means the scalar extension $V \otimes_{f} k$, where $f: k \rightarrow k, \lambda \mapsto \lambda^{p^{*}}$. Inductively $V^{(p)}=$ $\left(V^{\left(p^{r-1}\right)}\right)^{(p)}$.

0.1. Concerning coalgebras and Hopf algebras we freely use the notation and the terminology of Sweedler [6]. The structure maps of a $k$-coalgebra $C$ will generally be denoted by $\Delta: C \rightarrow C \otimes C$ and $\epsilon: C \rightarrow k . C^{+}$means $\operatorname{Ker}(\epsilon)$. The "sigma" notation $\Delta(x)=\Sigma_{(x)} x_{(1)} \otimes x_{(2)}$ for $x \in C$ will be used. The $k$-coalgebra $C$ is irreducible if any two nonzero subcoalgebras of $C$ have nonzero intersection $[6, \$ 8.0]$. A maximal irreducible subcoalgebra of $C$ is called an irreducible component of $C$. The k-coalgebra $C$ is cocommutative if $\Sigma_{(x)} x_{(1)} \otimes x_{(2)}=\Sigma_{(x)} x_{(2)} \otimes x_{(1)}$ for all $x \in C$.

The dual space $C^{*}$ becomes a $k$-algebra which is called the dual algebra of $C$, if the product is defined by $f * g=(f \otimes g) \circ \Delta$. It is commutative if $C$ is cocommutative.

Conversely, for each $k$-algebra $A$, the dual coalgebra $A^{0}$ is defined to be $\lim _{I}(A / I)^{*}$, where $I$ runs through all the cofinite twosided ideals of $A$ $[6, \$ 6.0]$. The functors $C \mapsto C^{*}$ and $A \mapsto A^{0}$ are adjoint to each other in the sense:

$$
A \lg _{k}\left(A, C^{*}\right) \simeq \operatorname{Coalg}_{k}\left(C, A^{9}\right)
$$

A subspace $I$ of a $k$-coalgebra $C$ is a coideal if $\epsilon(I)=0$ and $\Delta(I) \subset I \otimes$ $C+C \otimes I$. The quotient space $C / I$ then has a natural coalgebra structure. If $f: C \rightarrow D$ is a homomorphism of $k$-coalgebras, then the kemel $\operatorname{Ker}(f)$ is a coideal of $C$ and the map $f$ factors through $C \rightarrow C / \operatorname{Ker}(f)$.

The structure maps of a Hopf algebra $H$ over $k$ will generally be denoted by $\Delta: H \rightarrow H \otimes H, m: H \otimes H \rightarrow H, \epsilon: H \rightarrow k, u: k \rightarrow H$ and $S: H \rightarrow H$ (the antipode). The dual coalgebra $H^{0}$ of the $k$-algebra $H$ is a subalgebra of the dual algebra $H^{*}$ of the $k$-coalgebra $H$ and is stable under the map ${ }^{t} S$ : $H^{*} \rightarrow H^{*}, f \mapsto f \circ S$. The induced algebra structure makes $H^{0}$ a Hopf algebra with the antipode ${ }^{t} S \mid H^{0}$, called the dual Hopf algebra of $H[6, \S 6.2]$. The functor $H \mapsto H^{0}$ is selfadjoint in the following sense:

$$
\operatorname{Hopf}_{k}\left(H, K^{0}\right) \simeq \operatorname{Hopf}_{k}\left(K, H^{0}\right)
$$

for all $k$-Hopf algebras $H$ and $K$.

The bracket product in a $k$-Hopf algebra $H$ is defined by 


$$
[x, y]=\sum_{(x, y)} x_{(1)} y_{(1)} S\left(x_{(2)}\right) S\left(y_{(2)}\right)
$$

for $x, y \in H$. If $K$ and $J$ are sub-Hopf algebras of $H,[K, J]$ denotes the sub. algebra of $H$ generated by the elements $[x, y]$ with $x \in K$ and $y \in J$. If $H$ is cocommutative, this is a subbialgebra of $H$.

A subspace $I$ of $H$ is a Hopf ideal if it is a coideal and a twosided ideal of $H$ and $S(I) \subset I$. The quotient space $H / I$ then has a natural Hopf algebra structure. If $f: H \rightarrow H^{\prime}$ is a homomorphism of Hopf algebras, then the kernel $\operatorname{Ker}(f)$ is a Hopf ideal of $H$ and the map $f$ factors through $H \rightarrow H / I$.

If $K$ is a sub-Hopf algebra of $H$, then $\mathrm{HK}^{+}$is a coideal of $H$. The quotient coalgebra $H / H K^{+}$is denoted by $H / / K$. For each coideal $I$ of $H$, we put $l(I)=\{x \in H \mid \Delta(x)-1 \otimes x \in I \otimes H\}$. A sub-Hopf algebra $K$ of $H$ is normal if $\Sigma_{(x)} x_{(1)} y S\left(x_{(2)}\right) \in K$ for all $x \in H$ and $y \in K$ or equivalently if $[H, K] \subset$ $K$. A Hopf ideal $J$ of $H$ is normal if $\Sigma_{(x)} x_{(1)} S\left(x_{(3)}\right) \otimes x_{(2)} \in H \otimes J$ for all $x \in J$. In some cases we have a "bijective correspondence" between some class of sub-Hopf algebras and some class of coideals of $H$ as follows:

0.1.1. Proposition ([7] or [DG, III, $\left.\left.\$ 3, \mathrm{n}^{\circ} 7\right]\right)$. Let $H$ be a commutative Hopf algebra.

(a) If $K$ is a sub-Hopf algebra of $H$, then $H$ is faithfully flat over $K$ and $I=H K^{+}$is a normal Hopf ideal of $H$. If $H$ is finitely generated, then so is $K$.

(b) If $I$ is a normal Hopf ideal of $H$, then $l(I)$ is a sub-Hopf algebra of $H$.

(c) The correspondences $K \mapsto H K^{+}$and $I \mapsto l(I)$ establish a bijection between the sets of sub.Hopf algebras and of normal Hopf ideals of $H$.

0.1.2. Proposition $\left[\mathrm{T}_{\mathrm{II}}, 5.4 .2 .1,4.2 .2 .1,5.4 .2 .5,5.4 .3 .7\right]$. Let $H$ be a cocommutative Hopf algebra.

(a) If $K$ is a sub-Hopf algebra of $H$, then $H$ is a left and a right faithfully flat $K$-module [7] and an injective cogenerator in the category of right H//K-comodules. $\mathrm{HK}^{+}$is clearly a coideal and a left ideal of $H$.

(b) If $I$ is a coideal and a left ideal of $H$, then $l(I)$ is a sub-Hopf algebra of $H$.

(c) The correspondences $K \mapsto H K^{+}$and $I \mapsto l(I)$ establish a bijection between the sets of sub-Hopf algebras of $H$ and of coideal-left-ideals of $H$.

(d) The coideal $\mathrm{HK}^{+}$is a Hopf ideal of $H$ if and only if $K$ is normal in $H$. In particular the bijective correspondence of (c) induces a bijection between the sets of normal sub-Hopf algebras of $H$ and of Hopf ideals of $H$.

If $f: H \rightarrow H^{\prime}$ is a homomorphism of Hopf algebras, the Hopf kernel Hopf-ker $(f)$ is defined to be the largest sub-Hopf algebra $K$ of $H$ with $K^{+} \mathrm{C}$ $\operatorname{Ker}(f)$. This equals $l(\operatorname{Ker}(f))$ when $H$ is cocommutative or when $H$ is com- 
mutative and the Hopf ideal $\operatorname{Ker}(f)$ is normal in $H$.

Suppose $p>0$. The Verschiebung map of a cocommutative k-coalgebra $C$, $V_{C}: C \rightarrow C^{(p)}$ is a unique $k$-coalgebra map such that the composite

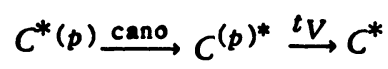

sends each element $X \otimes \lambda$ (with $X \in C^{*}$ and $\lambda \in k$ ) to $X^{p} \lambda\left[\mathrm{T}_{1}, 1.9 .1\right]$, [ $\mathrm{T}_{\mathrm{II}}$, 5.5.3.1]. If $H$ is a cocommutative Hopf algebra, the Verschiebung map $V_{H}: H \rightarrow H^{(p)}$ is a Hopf algebra map.

0.2. Conceming the basic theory of algebraic schemes and algebraic groups we refer the reader to [DG]. Here we briefly recall some of the fundamental relations between affine algebraic group schemes and commutative Hopf algebras.

A $k$-group scheme $B$ is affine algebraic if it is represented by some finitely generated commutative Hopf algebra $A$, that is $B=\operatorname{Spec}(A)$. The Hopf algebra $A$, which is uniquely determined by $B$, is denoted by $O(\mathbb{B})$. The following well-known relations between $B \leftrightarrow A$ are of particular importance:

$\mathbb{B}$ is smooth $\rightarrow A^{(p)}$ is reduced,

$B$ is connected $\rightarrow A$ has no idempotents other than 0 and 1 ,

(B) is unipotent $\Leftrightarrow A$ is irreducible as a coalgebra,

(B) is finite $\Rightarrow[A: k]<\infty$,

$\mathbb{B}$ is etale $\Rightarrow A$ is a finite product of finite separable extensions of $k$.

The additive and the multiplicative $k$-group schemes $\theta_{a}$ and $B_{m}$ are defined by

$$
\begin{array}{ll}
\otimes_{a}=\operatorname{Spec}(k[T]), & \Delta(T)=T \otimes 1+1 \otimes T, \\
B_{m}=\operatorname{Spec}\left(k\left[X, X^{-1}\right]\right), & \Delta(X)=X \otimes X .
\end{array}
$$

For each finitely generated abelian group $\Gamma$, the diagonalizable $k$-group scheme $\mathscr{S}(\Gamma)$ is defined by

$$
D(\Gamma)=\operatorname{Spec}(k[\Gamma]), \quad \Delta(y)=y \otimes y \quad \text { for all } y \in \Gamma .
$$

If $K / k$ is an extension of fields, the scalar extension $\& \otimes_{k} K$, where $B=\operatorname{Spec}(A)$, is $\operatorname{Spec}_{K}\left(A Q_{k} K\right)$.

The $k$-group schemes we shall treat are not necessarily smooth. In particular "finite connected" k-group schemes as follow will be taken into our consideration also:

$$
\begin{aligned}
q^{\alpha_{k}}=\operatorname{Spec}\left(k[T] / T^{q}\right), & \Delta(T)=T \otimes 1+1 \otimes T, \\
q^{\mu_{k}}=\operatorname{Spec}\left(k[X] /\left(X^{q}-1\right)\right), & \Delta(X)=X \otimes X,
\end{aligned}
$$

where $q=$ some power of $p^{*}$. The terms such as kernel, center, quotient, etc. 
should be always interpreted in the sense of affine algebraic $k$-group schemes (not in the sense of $\bar{k}$-rational points).

Let $B=\operatorname{Spec}(A)$ be an affine algebraic $k$-group scheme. The $k$-group scheme of the form $B=\operatorname{Spec}(B)$ with $B$ a sub-Hopf algebra of $A$, is called a quotient k-group scheme of $\$$. The $k$-group scheme of the form $\mathscr{F}_{2}=$ Spec $(A / I)$ with $I$ a Hopf ideal of $A$, is called a closed subgroup scheme of (P. The closed subgroup scheme $\mathscr{F}$ is normal in $(S)$ if so is the Hopf ideal $I$ in the sense of $\$ 0.1$. It follows from 0.1.1 (c) that there is a natural bijective correspondence between the sets of normal closed subgroup schemes and of quotient group schemes of $B$. The quotient group scheme of $B$ associated with a normal closed subgroup scheme $\Re$ of $B$ is denoted by $\mathbb{A} \Re$. If $\mathrm{F}: B \rightarrow \mathbb{B}^{\prime}$ is a homomorphism of affine algebraic $k$-group schemes, then the normal closed subgroup scheme of $B$ corresponding to the image of the induced Hopf algebra map $O(f): O\left(B^{\prime}\right) \rightarrow O(B)=A$, which is a sub-Hopf algebra of $A$, is called the kernel of $f$ and denoted by $\operatorname{Rex}(\mathcal{F})$. The map $F$ factors through $B \rightarrow B / \AA \operatorname{ex}(\Phi)$, via which the quotient group scheme $B / \AA \operatorname{ex}(\uparrow)$ can be viewed as a closed subgroup scheme of $C^{\prime}$ naturally. The map $F$ is an epimorphism (resp. a monomorphism) if $B / \operatorname{Rex}(F) \simeq B^{\prime}$ (resp. $\operatorname{Rex}(\mathcal{F})=$ (e)) or equivalently if the Hopf algebra map $\theta(\eta)$ is injective (resp. surjective). A sequence $1 \rightarrow \Re \stackrel{i}{\rightarrow}(\mathbb{P}) \mathbb{B}$ " $\rightarrow 1$ of affine algebraic $k$-group schemes is exact if $i: \Re \simeq \Re e x(p)$ and $p: B / \Re \simeq B "$.

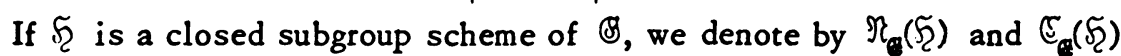
the normalizer and the centralizer of $\mathcal{F}_{2}$ in $\$$ respectively [DG, II, $\$ 1, n^{\circ} 3$ ]. They are closed subgroup schemes of $\&[D G, I I, \$ 1,3.7]$. In particular so is the centre cent(B).

The derived group [B, $B$ ] is defined to be the kemel of the projection $B \rightarrow$ Sped $(B)$, where $B$ denotes the largest cocommutative sub-Hopf algebra of $\Theta(B)$. If $B$ is smooth, this definition coincides with [DG, II, $\$ 5,4.8$ ].

Let $\&$ be a connected smooth affine algebraic $k$-group scheme. When $k$ is perfect, we define the radical $x a b(\mathbb{B})$ (resp. the unipotent radical $\mathbb{B}{ }_{u}$ ) of $\mathbb{B}$ to be the largest normal connected smooth solvable (resp. unipotent) closed subgroup scheme of $\$ 8$. If $\bar{k}$ denotes the algebraic closure of $k$, then rab(\&) $\otimes_{k} \bar{k}$ (resp. $\otimes_{u} \otimes_{k} \bar{k}$ ) is the radical (resp. the unipotent radical) of \& $\otimes_{k} \bar{k}$ in the usual sense. The smooth connected $k$-group scheme $\&$ is semisimple (resp. reductive) if $\operatorname{rab}(\&)=(e)$ (resp. $\&_{u}=(e)$ ).

Suppose $p>0$ in the rest of this $\$ 0.2$. The Frobenius map $₹: B \rightarrow \mathbb{B}(p)$ of an affine algebraic $k$-group scheme $\mathbb{B}=\operatorname{Spec}(A)$ corresponds to the Hopf algebra map $A^{(p)} \rightarrow A, a \otimes \lambda \mapsto a^{p} \lambda$ with $a \in A$ and $\lambda \epsilon k$. The k-group 
scheme $\mathbb{F}$ is smooth (resp. etale) if and only if the Frobenius map $\mathscr{F}$ is an epimorphism (resp. a monomorphism).

When $(8)$ is commutative (or equivalently if $\Theta(B)=A$ is cocommutative), the Verschiebung map of $\&, \mathbb{P}_{\mathbb{B}}:(B) \rightarrow \&\left[D G, I V, \$ 3, n^{\circ} 6\right]$ corresponds to the Verschiebung map of $A, V_{A}: A \rightarrow A^{(p)}$ (defined in $\$ 0.1$ ).

Let $k[F]$ denote the noncommutative polynomial ring over $k$ defined by $F \lambda=\lambda^{p} F$ for all $\lambda \in k$. The category of commutative affine algebraic $k$-group schemes killed by the Verschiebung map is antiequivalent to the category of finitely generated left $k[F]$-modules $[D G, I V, \S 3,6.6]$. We denote by $\mathfrak{U}(M)$ the $k$-group scheme determined by a left $k[F]$-module $M$. If we view $M$ as a commutative $p$-Lie algebra over $k, U^{[p]}(M)$, the universal enveloping algebra of $M$, has a unique Hopf algebra structure having $M$ as primitive elements (i.e., $\Delta(x)=x \otimes 1+1 \otimes x$ for all $x \in M$ ) and we have $\mathfrak{U}(M)=\operatorname{Spec}\left(U^{[p]}(M)\right.$ ) with this Hopf algebra structure. We have the following equivalence relations:

$\mathfrak{u}(M)$ is smooth $\Leftrightarrow 0 \rightarrow M^{(p)} \stackrel{F}{\rightarrow} M$,

$\mathfrak{u}(M)$ is finite $\Leftrightarrow[M: k]<\infty$,

$\mathfrak{U}(M)$ is etale $\Leftrightarrow M=k F M$,

$\mathfrak{U}(M)$ is connected $\Leftrightarrow$ each torsion element of $M$ is killed by some power of $F$.

If $k$ is algebraically closed, we have further

$\mathfrak{U}(M)$ is etale $\Leftrightarrow M \simeq(k[F] / F-1)^{s}$,

$\mathfrak{U}(M)$ is connected smooth $\Leftrightarrow M \simeq k[F]^{r}$

[DG, IV, $\$ 3,6.11]$.

0.3. As a final preliminary, we briefly recall the theroy of hyperalgebras developed in $\left[\mathrm{T}_{\mathrm{I}}\right],\left[\mathrm{T}_{\mathrm{II}}\right]$.

A byperalgebra means an irreducible cocommutative Hopf algebra. The Lie algebra $\mathrm{Lie}(J)$ of a hyperalgebra $J$ is by definition the primitive elements of $J, P(J)=\{x \in J \mid \Delta(x)=x \otimes 1+1 \otimes x\}$. When $\mathrm{Lie}(J)$ is finite dimensional, $J$ is called of finite type. If so is $J$, the dual algebra $J^{*}$ is noetherian $\left[\mathrm{T}_{1}\right.$, 1.4. 1] and has a finite Krull dimension which cannot exceed [ $\mathrm{Lie}(J): k]$. We define the Krull dimension $\operatorname{dim}(J)$ to be the Krull dimension of $J^{*}\left[\mathrm{~T}_{1}, 1.4 .4\right]$. When the equality $\operatorname{dim} J=[\mathrm{Lie}(J): k]$ holds, the finite type hyperalgebra $J$ is called smooth or of Birkboff-Witt type $\left[\mathrm{T}_{1}, 1.6 .1\right]$. This is equivalent to saying $J \simeq B(U)$ as a coalgebra, for some finite dimensional $k$-vector space $U$ with the notation of $[6, \$ 12.2]$. If $p=0$ all hyperalgebras are smooth and if $p>0, J$ is smooth if and only if the Verschiebung map $V_{J}: J \rightarrow J^{(p)}$ is surjective $\left[T_{I}, 1.9 .4\right]$. From this it follows easily that an arbitrary finite type hyperalgebra $J$ has the largest smooth subhyperalgebra $J_{s m}$ called the smooth part of $J$ if $k$ is perfect $\left[T_{I}, 1.9 .5\right]$. Then the quotient coalgebra $J / / J_{s m}$ is 
finite dimensional over $k$ and we have $\operatorname{dim}(J)=\operatorname{dim}\left(J_{\mathrm{sm}}\right)=\left[\operatorname{Lie}\left(J_{\mathrm{sm}}\right): k\right]$ $\left[\mathrm{T}_{\mathrm{II}}\right.$, 5.5.3.8].

Among other things very important and useful is the fact that if $p>0$ any finite type hyperalgebra $J$ is the union of its finite dimensional normal subhyperalgebras $\left[\mathrm{T}_{\mathrm{II}}, 5.5 .3 .7,5.5 .3 .9\right]$.

Let $B$ be a locally algebraic (not necessarily affine) $k$-group scheme [DG, II, $\$ 5]$. Let $\theta_{e}$ denote the stalk over the unit $e$ of the structure sheaf $O_{0}$. The multiplication $\& \times B \rightarrow$ defines naturally a multiplication of the dual coalgebra $\left(\Theta_{e}\right)^{0}$ and makes it a finite type hyperalgebra $\left[\mathrm{T}_{1}, 3.1 .4,3.3 .1\right]$ which is denoted by hy(B) and called the hyperalgebra of $\mathbb{B}$.

If $\&$ is affine, then hy(\&) coincides with the irreducible component containing 1 of the dual Hopf algebra $\Theta(\&)^{0}$. In particular, since the functor $H$ $\mapsto H^{0}$ is selfadjoint $(\$ 0.1)$, the inclusion hy(\&) $\hookrightarrow \Theta(\&)^{0}$ corresponds to a natural Hopf algebra map $O(B) \rightarrow$ hy(B) ${ }^{0}$ which is not necessarily injective.

A homomorphism of locally algebraic $k$-group schemes $f: \& \rightarrow \mathbb{C}^{\prime}$ induces clearly a homomorphism of hyperalgebras hy( $f):$ hy( $(\&) \rightarrow$ hy(\& $)$. If $(8)$ is affine, it is easy to see that the natural map $\theta(\mathbb{B}) \rightarrow$ hy(B) ${ }^{0}$ is bijective if and only if the natural map $\mathrm{Hom}_{k-g r}\left(\&, \mathbb{B}^{\prime}\right) \rightarrow \mathrm{Hopf}_{k}\left(\mathrm{hy}(\mathbb{B}), \mathrm{hy}\left(\mathbb{B}^{\prime}\right)\right.$ ) which sends each map $f$ to the induced map hy( $P$ is bijective for all affine algebraic $k$-group schemes $B^{\prime}$.

The functor $B \mapsto$ hy( $(\mathbb{S})$ has many interesting properties which are similar and reduce to the properties of the functor $B \operatorname{Lie}(\mathbb{B})$ in case $p=0$ :

0.3.1. Proposition. Let \& be a locally algebraic k-group scheme.

(a) $\left[\mathrm{T}_{\mathrm{I}}, 3.1 .8,3.3 .1\right]$. The Lie algebra of $\$$, Lie(\&) [DG, II, $\left.\$ 4, \mathrm{n}^{\circ} 1\right]$, equals Lie(hy(B)). The dimension of $\&, \operatorname{dim} \&[\mathrm{DG}, \mathrm{II}, \$ 5,1.3]$, equals $\operatorname{dim}(h y($ (E)).

(b) $\left[\mathrm{T}_{1}, 3.1 .7\right]$. If $K / k$ is an arbitrary extension of fields, then hy(\&) $\otimes_{k}$ $K$ equals the K-hyperalgebra hy ${ }_{K}\left(\& \otimes_{k} K\right)$ of the locally algebraic $K \cdot$ group scheme $8 \otimes_{k} K$.

(c) $\left[\mathrm{T}_{1}, 2.2 .9\right]$. If $p>0$, the Frobenius map $\mathfrak{F}_{\odot}: B \rightarrow B(p)$ induces the Verschiebung map $V:$ hy(\&) $\rightarrow$ hy(\&) $)^{(p)}$.

(d) $\left[\mathrm{T}_{\mathrm{I}}, 3.3 .5,3.3 .11\right]$. \& is smooth if and only if hy(\&) is. When $k$ is perfect, let $\&_{\text {red }}$ denote the reduced part of $\&[\mathrm{DG}, \mathrm{II}, \$ 5,2.3]$. Then the byperalgebra hy(B $\left(\mathbb{R}_{\text {red }}\right)$ equals the smooth part hy(B) $)_{\mathrm{sm}}$ of hy(B).

(e) $\left[\mathrm{T}_{1}, 3.3 .3\right]$. \& is etale if and only if hy(B) $=k$.

(f) $\left[\mathrm{T}_{1}, 3.3 .6\right]$. A subgroup scheme $\mathfrak{S}_{2}$ of $B$ is open if and only if by( $\left(\mathfrak{S}_{2}\right)$ $=$ hy(\&). In particular if $\&$ is connected, then $\mathscr{F}_{2}=\mathbb{B}$ if and only if hy(F) = hy(B). 
(g) If 8 is affine, the canonical map $O(B) \rightarrow$ hy(B) ${ }^{0}$ is injective if and only if $(8)$ is connected.

(h) If $p>0$ and if $(8$ is affine connected, then $\&$ is unipotent if and only if each element of hy(\&) ${ }^{+}$is nilpotent.

0.3.2. Proposition. Let $\mathrm{f}: B \rightarrow \mathbb{B}^{\prime}$ be a homomorphism of locally algebraic k-group schemes.

(a) $\left[\mathrm{T}_{1}, 3.1 .5\right]$. The hyperalgebra of the kemel $\mathrm{Rex}(\mathrm{f})$ equals the Hopf kemel of hy( $f)$. In particular if $F$ is a monomorphism, then hy( $f)$ is injective.

(b) $\left[\mathrm{T}_{1}, 3.3 .2\right]$. If both $\&$ and $\&{ }^{\prime}$ are algebraic, there is a unique closed subgroup scheme $F(\&))^{\sim}$ of $\mathbb{S}^{\prime}$, called the image-subgroup of $F$, such that the induced map $\mathrm{F}: \mathrm{BS} \rightarrow \mathrm{F}(\mathbb{B})^{\sim}$ is faithfully flat [DG, III, $\left.\$ 3,5.2,2.6, \mathrm{II}, \$ 5.5 .1\right]$. Then we have

$$
\text { hy }(F(\&))=\operatorname{Im}(\text { hy }(F))=\text { hy }(\&) / / \operatorname{Hopf}-k e r(h y(F)) \text {. }
$$

(c) $\left[T_{I}, 3.3 .3\right]$. The map $F$ is nonramified [DG, $\left.I, \$ 4,3.2\right]$ if and only if hy $(P)$ is injective.

(d) $\left[\mathrm{T}_{\mathrm{I}}, 3.3 .4\right]$. The map $\mathrm{F}$ is flat if and only if hy( $\left.f\right)$ is surjective.

(e) $\left[\mathrm{T}_{\mathrm{I}}, 3.3 .7\right]$. If $\mathbb{B}^{\prime}$ is connected and $B$ is algebraic, then $\left.\mathrm{f(B)}\right)^{2}=$ $B^{\prime}$ if and only if hy( $\left.\mathcal{F}\right)$ is surjective.

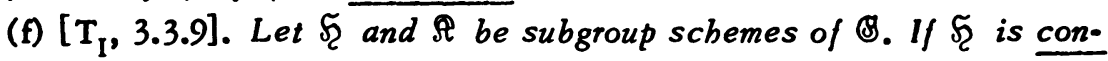
nected, then $\mathscr{S}_{2} \subset \Omega$ if and only if hy( $\left.\mathfrak{F}_{2}\right) \subset \mathrm{hy}(\Re)$. In particular the correspondence $\mathcal{F}_{\mathrm{H}} \mapsto \mathrm{hy}(\mathfrak{F})$ from the set of connected (and bence closed) subgroup schemes of $B$ into the set of subbyperalgebras of hy(B) is injective.

(g) $\left[\mathrm{T}_{\mathrm{I}}, 3.3 .10\right]$. Let $\mathrm{f}^{\prime}: \mathbb{B} \rightarrow \mathbb{B}^{\prime}$ be another homomorphism. If $\mathbb{B}$ is connected (and hence algebraic), then $F=F^{\prime}$ if and only if hy $(F)=\operatorname{hy}\left(F^{\prime}\right)$.

Let 8 be a locally algebraic $k$-group scheme. The inner automorphism action $B \times B \rightarrow B,(g, h) \mapsto g h g^{-1}$ induces a linear representation $\mathscr{P D}: \mathbb{B} \rightarrow$ $\mathbb{B}\left\{\right.$ (hy(\&)), called the adjoint representation of $\&\left[\mathrm{~T}_{1}, 3.1 .6,3.4 .13\right]$. We view as usual each $k$-group scheme as a $k$-group functor, i.e., a functor from the category $M_{k}$ of $k \cdot$ models to the category of groups [DG, $\left.I, \$ 1, n^{\circ} 4\right]$.

0.3.3. Proposition. Let $\sqrt{2}$ be a closed subgroup scheme of a locally algebraic k-group scheme $\mathbb{B}$. $\Re_{\mathrm{g}}\left(\mathfrak{F}_{2}\right)$ and $\mathcal{E}_{\mathrm{e}}\left(\xi_{2}\right)$ denote the normalizer and the centralizer of $5 \mathcal{2}$ in 8 .

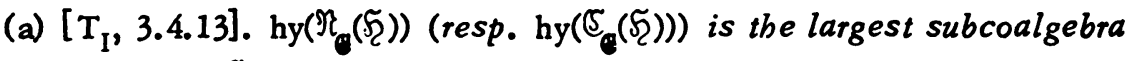
$C$ (resp. D) of hy(\&) satisfying

$$
\sum_{(c)} \mathfrak{M S}(h)\left(c_{(1)}\right) S\left(c_{(2)}\right) \in R \otimes h y(5)
$$

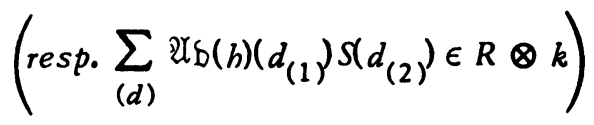


for all $R \in \mathrm{M}_{k}, b \in \mathcal{S}_{2}(R)$ and $c \in C$ (resp. $d \in D$ ), where $S$ denotes the antipode of hy(B).

(b) $\left[\mathrm{T}_{\mathrm{I}}, 3.4 .15\right]$. If \& 8 is connected (and hence algebraic) then fु is nor mal (resp. central) in $\&$ if and only if

$\sum_{(a)} \mathscr{P D}(b)\left(a_{(1)}\right) S\left(a_{(2)}\right) \in R \otimes h y\left(S_{2}\right)$

$$
\left(\text { resp. } \sum_{(a)} \operatorname{Pb}(h)\left(a_{(1)}\right) S\left(a_{(2)}\right) \in R \otimes k\right)
$$

for all $R \in \mathrm{M}_{k}, b \in \mathcal{S}_{2}(R)$ and $a \in \mathrm{hy}(\mathbb{B})$.

(c) $\left[\mathrm{T}_{\mathrm{I}}, 3.4 \mathrm{a} .5\right]$. If $\mathfrak{S}_{2}$ is connected, then $\mathfrak{F}_{2}$ is normal (resp. central) in

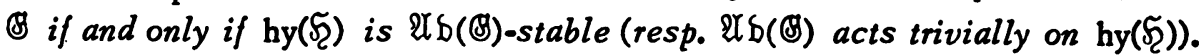

(d) $\left[\mathrm{T}_{\mathrm{I}}, 3.4 .15\right]$. If $\mathfrak{F}_{2}$ and $\&$ are both connected, then $\mathscr{S C}_{2}$ is normal (resp. central) in $\&$ if and only if so is the subbyperalgebra hy(5ૃ) in hy(\&). (A sub-Hopf algebra $J$ of a Hopf algebra $H$ is central if $[H, J]=k$.)

Let $\&$ be a locally algebraic $k$-group scheme. A subhyperalgebra $J$ of hy(B) is algebraic (or closed) if there is a subgroup scheme $\mathscr{F}$ of $\mathbb{B}$ with $J=$ hy(f) $)$. It follows from $0.3 .2(f)$ that we can take then a unique connected (and

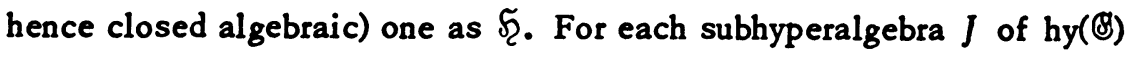
there is a unique smallest algebraic subhyperalgebra $A(J)$ of hy(B) containing $J\left[T_{1}, \$ 3.6\right]$ called the algebraic hull of $J$.

0.3.4. Proposition. Let \& be a locally algebraic $k$-group scheme and $J$ a subhyperalgebra of hy(8).

(a) If $[J: k]<\infty$, then $J$ is closed.

(b) Let $K$ be a subbyperalgebra of J. If $\mathrm{J} / / \mathrm{K}$ is finite dimensional, then $K$ is closed in hy $(G)$ if and only if so is $J$.

(c) Let $l / k$ be an arbitrary extension of fields. Then $J$ is closed in hy(\&) if and only if so is $J \otimes l$ in hy(\$) $\otimes l=\mathrm{hy}_{l}(\mathbb{Q})$.

(d) $\left[\mathrm{T}_{\mathrm{I}}, 3.6 .2\right]$. Let $A$ be a subalgebra and $C$ a subcoalgebra of hy(B) such that $A C \subset C$. If $[J, C] \subset A$, then $[A(J), C] \subset A$.

(e) $\left[\mathrm{T}_{\mathrm{I}}, 3.6 .2\right]$. Let $K$ be a subbyperalgebra of J. If $K$ is normal in $J$, then so is $K$ in $A(J)$.

(f) $\left[\mathrm{T}_{\mathrm{I}}, 3.6 .3\right]$. We have $[J, J]=[A(J), A(J)]$ (which is closed in hy(B) by the following $(\mathrm{g})$ ). In particular the quotient byperalgebra $A(J) / / J$ is abelian.

(g) If $J_{1}$ and $J_{2}$ are closed subhyperalgebras of hy(), then the commutator subhyperalgebra $\left[J_{1}, J_{2}\right]$ is also closed in hy(\$). 

hy(\&)].

(h) $\left[\mathrm{T}_{\mathrm{I}}, 3.5 .6\right]$. If $\&$ is connected smooth, then $\mathrm{hy}([\&, \mathbb{B}])=[\mathrm{hy}(\mathbb{8})$,

(i) If \& is connected affine, then hy([\&, \&] $)=[$ hy(B), hy(\&) $]$.

The proof of (a), (b), (c), ( $f),(g)$ and (i) above will be published elsewhere. In particular if $\mathscr{S}$ and $\Omega$ are connected subgroup schemes of a locally algebraic $k$-group scheme $\mathbb{B}$, we can define the commutator subgroup $[\mathfrak{L}, \Omega]$ to be the unique connected subgroup of $\mathbb{S}$ with hy $\left(\left[\mathcal{F}_{2}, \mathbb{R}\right]\right)=[$ hy $(\mathscr{S})$, hy $(\mathbb{R})]$. This definition generalizes [DG, II, \$5, 4.9].

1. The (SC) (or (SC) $)_{p} k$-group schemes. We have defined in the introduction the concepts of etale group covering, universal group covering, (SC) or $(\mathrm{SC})_{p} k$-group scheme, etc.

1.1. Proposition. Let $\&$ and $\mathscr{F}_{\text {be connected affine algebraic } k \cdot g r o u p}$ schemes and $\eta: \mathscr{F}_{\rightarrow} \rightarrow$ \& a homomorphism of k-group schemes. Then $(\mathcal{F}, \eta)$ is an etale group covering of $\&$ if and only if hy( $\eta): h y\left(F_{2}\right) \simeq$ hy(B).

This follows directly from $0.3 .2(c)$ and (e).

1.2. Proposition. Let \&S be a connected affine algebraic k-group scheme and $\AA$ a normal connected closed subgroup scheme of $\&$. (a) If $\&$ is (SC) (resp. $(S C)_{p}$ ), then so is $\mathbb{R} / \Omega$. (b) If $\mathbb{S} / \Omega$ and $\Omega$ are both (SC) (resp. (SC) $)$, then so is \&.

The proof is easy.

1.3. Remark. All finite connected $k$-group schemes are clearly (SC).

1.4. Remark. The multiplicative group $\&_{m}$ is not $(\mathrm{SC})_{p}$, in view of the canonical extension $1 \rightarrow{ }_{n} \mu \rightarrow \mathbb{B}_{m} \rightarrow \mathbb{B}_{m} \rightarrow 1$ for all $n$ relatively prime to $p^{*}$. More generally if $\mathscr{F}_{\mathcal{C}}$ is a $k$-torus, that is $\mathscr{S}_{\mathcal{C}} \otimes \bar{k} \simeq\left(\otimes{ }_{m} \otimes \bar{k}\right)^{r}$ for some $r \in \mathrm{N}$, then $\mathfrak{F}$ is not $(\mathrm{SC})_{p}$ unless $r=0$. Indeed if $\mathbb{R}$ denotes the kemel of the morphism $\mathscr{F}_{\rightarrow} \rightarrow F_{2}, x \mapsto x^{n}$, then $\Omega \otimes \bar{k} \simeq\left({ }_{n} \mu \otimes \bar{k}\right)^{r}$ clearly and so $\Re$ is finite etale with the order $n^{r}$ which is prime to $p^{*}$ if $n$ is so chosen. The additive group $B_{a}$ is not (SC) when $p>0$, in view of the Artin-Schreier extension

$$
1 \rightarrow\left(\mathrm{Z} / \mathrm{pZ}_{k} \rightarrow \stackrel{\mathbb{B}-1}{\longrightarrow} \stackrel{\mathbb{P}}{\mathbb{P}_{a}} \rightarrow 1\right.
$$

where $\mathfrak{F}$ denotes the Frobenius map.

In general, each affine algebraic $k$-group scheme 8 has a normal closed connected finite subgroup scheme $\Re$ such that $\& / \Re$ is smooth [DG, III, $\$ 3$, 6. 10]. Since $\Re$ is always (SC), it follows that $B$ is (SC) (resp. $(\mathrm{SC})_{p}$ ) if and 
only if $\mathbb{A} / \Re$ is. If $\mathbb{A} / \Re$ has a $(p-)$ universal group covering, then pulling it back along $B \rightarrow A / \Re$, we obtain a $(p-)$ universal group covering of $\&$. Hence all consideration concerning group coverings reduces to the case of smooth $k$-group schemes.

1.5. Proposition. Connected affine algebraic unipotent $k-g r o u p$ schemes are $(S C)_{p}$.

Proof. We shall consider the case $p>0$ first. Let $\&$ be connected unipotent $\neq(e)$. Since $B \neq[\mathbb{B}, \mathbb{B}]$, it suffices to prove that $\mathbb{B} /[\mathbb{B}, \mathbb{B}]$ is $(\mathrm{SC})_{p}$, by the induction hypothesis. Thus we can assume $B$ is commutative. Let

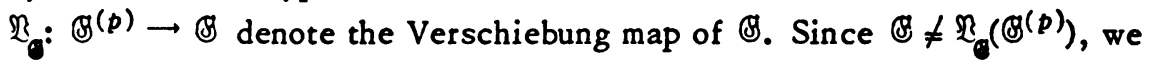
can assume that $\mathbb{B}_{\mathcal{C}}=0$, by the induction hypothesis again. There is then a finitely generated left $k[F]$-module $M$ with $B \simeq \mathfrak{U}(M)(\$ 0.2)$. Let $1 \rightarrow \Omega \rightarrow$ $\mathfrak{S} \rightarrow \mathfrak{U}(M) \rightarrow 1$ be a $p$-etale group covering of $\mathfrak{U}(M)$. Since hy( $(\mathcal{Z}) \simeq \operatorname{hy}(\mathfrak{l}(M))$, it follows from $0.3 .1(\mathrm{~h})$ that $\mathscr{F}$ is unipotent and from $0.3 .1(\mathrm{~g})$ that $\mathscr{F}_{2}$ is commutative. Since hy $\left(\mathbb{B}_{\mathscr{g}}\right)$ is trivial, it follows from $0.3 .2(\mathrm{~g})$ that $\mathfrak{B}_{\mathfrak{g}}=0$. In particular we have $\mathfrak{R}_{\mathbb{R}}=0$ and so $\mathbb{R} \mathfrak{M}(N)$ for some finite dimensional (over $k$ ) left $k[F]$-module $N$. The order of $\Omega\left(=\left[U^{[p]}(N): k\right]\right)$ is a power of $p$. But since the order of $\Re$ is relatively prime to $p$, it follows that $\Re=(e)$. Hence $\mathfrak{U}(M)$ is $(S C)_{p}$.

Next suppose that $p=0$. Since each affine algebraic unipotent $k$-group scheme has a central series of closed subgroup schemes each of whose quotients is isomorphic to $\mathbb{B}_{a}[\mathrm{DG}, \mathrm{IV}, \$ 2,3.9,4.1]$, it suffices to show that $\mathbb{A}_{a}$ is $(\mathrm{SC})_{0}$. But since any etale group covering of $\mathbb{B}_{a}$ is clearly 1-dimensional unipotent and hence isomorphic to $\mathbb{B}_{a}[D G, I V, \$ 2,2.10]$, the claim follows from the fact that $\mathbb{B}_{a}$ has no nontrivial etale subgroup scheme [DG, IV, $\$ 2$, 1.1]. Q.E.D.

1.6. Theorem. If $k$ is perfect, a connected smooth affine algebraic $k$ group scheme $\&$ is $(S C)_{p}$ if and only if the radical rab(\&) is unipotent and that $\bar{B}=B / \mathrm{xab}(\mathbb{B})$ is $(S C)$.

Proof. The 'if' part follows from Propositions 1.5 and 1.2. Suppose 8 is $(\mathrm{SC})_{p}$. Let $B_{u}$ denote the unipotent radical of $B$. Since $\bar{B}=B / B{ }_{u}$ is $(\mathrm{SC})_{p}$ and reductive, it follows that $\bar{B} /[\overline{\mathbb{B}}, \overline{\mathbb{B}}]$ is an $(\mathrm{SC})_{p}$ torus. But because any nontrivial $k$-torus is not $(\mathrm{SC})_{p}$, it follows that $\bar{B}=[\bar{F}, \bar{F}]$ or equivalently that $\bar{B}$ is semisimple. Since we show in $\$ 3$ that all connected (SC) $p$ semisimpel $k$-group schemes are (SC), it follows that $\bar{B}$ is (SC). Q.E.D.

1.7. Theorem. If $k$ is perfect, a connected smooth affine algebraic $k$. 
group scheme \& has a p-universal group covering if and only if the radical $\mathrm{rab}(\$)$ is unipotent.

Proof. The 'only if' part follows from Theorem 1.6. Suppose conversely that the radical $\mathrm{rab}(\mathbb{B})$ is unipotent. The semisimple $k$-group scheme $\bar{B}=$ $\mathbb{B} / \mathrm{ra \delta}(\mathbb{B})$ has a $p$-universal group covering $\overline{B^{*}}$ as we shall see in $\$ 3$. The pull-back $\mathbb{B}^{*}=\mathbb{B} \times_{\overline{\mathbb{S}}} \bar{B}^{*}$ then is a $p$-universal group covering of $\mathbb{B}$ by Theorem 1.6. Q.E.D.

1.8. Lemma. Let \& be a connected affine algebraic $k$-group scheme and consider the following three conditions:

(a) B is (SC).

(b) For each locally algebraic (not necessarily affine) k-group scheme $\mathfrak{F}$, the natural map

$$
\operatorname{Hom}_{k-g r}\left(\mathbb{B}, \tilde{S}_{2}\right) \rightarrow \mathrm{Hopf}_{k}(\mathrm{hy}(\mathbb{S}), \mathrm{hy}(\tilde{\tilde{C}})), \quad \text { I } \mapsto \mathrm{hy}(\mathcal{F})
$$

is bijective.

(c) The canonical map $\Theta(\mathbb{B}) \rightarrow \mathrm{hy}(\mathbb{B})^{0}$ is bijective.

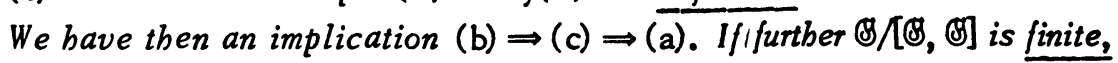
then $(\mathrm{a}) \Rightarrow(\mathrm{b})$.

Proof. We pointed out in $\$ 0.3$ that condition (c) is equivalent to the bijectivity of the maps of (b) for all affine algebraic $k$-group schemes $\mathfrak{F}_{\text {. In }}$ particular we have $(b) \Rightarrow(c)$. Let $\gamma: \bar{B} \rightarrow \&$ be an etale group covering. Since hy $(\gamma)$ is isomorphic, if (c) is valid, there is a unique homomorphism $\sigma: \& \rightarrow$ Fith $\operatorname{hy}(\sigma)=\operatorname{hy}(\gamma)^{-1}$. Then $\gamma \circ \sigma=\mathrm{id}$, because hy $(\gamma \circ \sigma)=$ id. Since $\sigma$ is an epimorphism of affine algebraic $k$-group schemes, it follows that $\sigma \circ \gamma$ $=$ id. Hence $\gamma$ is an isomorphism, so we have $(c) \Rightarrow(a)$. Finally suppose that $B$ is (SC) with $\& /[\&, \&]$ finite. Let $\mathscr{S}_{2}$ be an arbitrary locally algebraic $k$ group scheme. Let $\omega$ : hy(B) $\rightarrow$ hy $\left(F_{2}\right)$ be a homomorphism of hyperalgebras. The composite

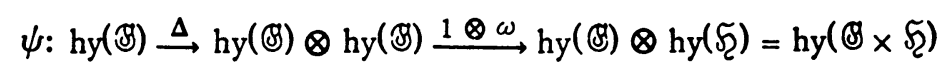

is an injective homomorphism of hyperalgebras. Put $\mathbb{\Omega}=\mathbb{B} \times \mathcal{F}, J=\operatorname{Im}(\psi)$ and $J^{\prime}=[J, J]$. Then $J^{\prime}$ is closed in hy( () by $0.3 .4(f)$ and $J / / J^{\prime} \simeq$ hy(ß/[\&, \&]) $(0.3 .2(b))$ is finite dimensional by assumption and hence $J$ is closed in hy $(\mathbb{B} \times \sqrt[5]{ })$ by $0.3 .4(\mathrm{~b})$. There exists a unique connected closed subgroup scheme $\mathbb{B}^{*}$ of $\mathbb{B} \times \sqrt{2}$ with hy( $\left.\mathbb{B}^{*}\right)=J$. The projection $\not x_{1}: \mathbb{B}^{*} \rightarrow \mathbb{B}$, which is then an etale group covering of $B$, is an isomorphism because $B$ is (SC). The composite

$$
\mathrm{F}: \stackrel{\left(p x_{1}\right)^{-1}}{\longrightarrow} \mathbb{B} * \stackrel{p x_{2}}{\longrightarrow} \mathcal{F}_{2}
$$


is easily seen to be a unique homomorphism of $k$-group schemes with hy $(\uparrow)=$ $\omega$. Q.E.D.

1.9. Theorem. If $k$ is perfect and $p>0$, a connected affine algebraic

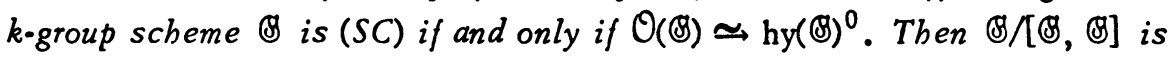
finite.

Proof. By virtue of Lemma 1.8 , it suffices to show that $\mathbb{B} /[\mathbb{B}, \mathbb{B}]$ is finite when $B$ is (SC). Suppose $B$ is (SC) and $p>0$. We can assume that $B$ is smooth. Let $B_{u}$ denote the unipotent radical of $\&$. Since $B / B{ }_{u}$ is semisimple (1.6), it follows that $B=[\&, \mathbb{B}]{ }_{u}$. Hence $\mathbb{B} /[B, \mathbb{B}]$ is (SC), unipotent and smooth. Since it has a central series of closed connected subgroups each of whose quotients is isomorphic with \& ${ }_{a}[D G, I V, \$ 2,3.9]$, it follows from Remark 1.4 that $B=[\&, \mathbb{B}]$. Q.E.D.

2. The case of Chevalley group schemes. In this section we assume $k$ to be algebraically closed and consider the problem of group coverings for connected semisimple $k$-group schemes. A connected smooth affine algebraic $k$-group scheme is semisimple if its radical is $(e)$. Let $\&$ be a connected semisimple $k$-group scheme with a maximal torus $\mathfrak{I}$. The character group $X=$ $X(\mathfrak{I})=\operatorname{Hom}_{k-g r}\left(\mathfrak{I}, \mathbb{S}_{m}\right)$ is a free Z-module of finite rank and has a natural root system $\nabla$ in it. For each root $a \in \nabla$, the associated coroot $a^{*}$ is uniquely detemined in the space $\hat{X}=\operatorname{Hom}_{Z}(X, Z)$. Let $\nabla^{*}$ denote the set of coroots $a^{*}, a \in \nabla$. We then have the following subgroups of $X_{Q}=X \otimes_{Z} \mathbf{Q}$ :

$$
X_{0}=\{\nabla\}_{Z} \subset X \subset X^{0}=\left\{\nabla^{*}\right\}_{Z}=\left\{x \in X_{Q} \mid\left\langle\nabla^{*}, x\right\rangle \subset Z\right. \text { Z }
$$

The group $X^{0}$ is called the weight module of $(X, \nabla)$. The quotient group $X^{0} / X_{0}$ is finite. See Iwahori [3, Vol. 2, p. 58] for the table of $X^{0} / X_{0}$ for the irreducible root systems. For any subgroup $Y$ between $X_{0}$ and $X^{0}$, the pair $(Y, \nabla)$ is a reduced root system, say, in the sense of Satake [5, p. 44] and if we identify $Y_{\mathrm{Q}}=X_{\mathrm{Q}}$, then $Y$ has the same coroot system as $X$ and we have $Y_{0}=X_{0}$ and $Y^{0}=X^{0}$.

Let $\mathrm{F}: A \rightarrow B$ ' be an "isogeny" of connected affine algebraic $k$-group schemes, by which we mean an epimorphism of affine algebraic $k$-group schemes whose kemel $\operatorname{Re} x(f)$ is a finite $k$-group scheme. If $B^{\prime}$ is smooth and $\operatorname{Rer}(\mathcal{P})$ is etale, then $B$ is smooth too. Suppose that $B$ and $B^{\prime}$ are both smooth. If one of $B$ and $B^{\prime}$ is reductive (resp. semisimple), then so is the other. Hence suppose that both $\mathbb{B}$ and $\mathbb{C}^{\prime}$ are semisimple. If $\mathfrak{T}$ is a maximal torus of $\mathbb{B}$, then $\mathfrak{T}^{\prime}=F(\mathfrak{T})$ is a maximal torus of $\mathbb{B}^{\prime}$ and the restricted isogeny $F: \mathfrak{I} \rightarrow \mathfrak{I}^{\prime}$ induces an injection of abelian groups ${ }^{t} \mathrm{~F}: X^{\prime}=X(\mathfrak{T}) \hookrightarrow$ $X=X(\stackrel{T}{\mathbb{x}})$. This is a special homomorphism in the following sense: 
(i) $\left[X:{ }^{t} f\left(X^{\prime}\right)\right]<\infty$.

(ii) If $\nabla$ and $\nabla^{\prime}$ denote the root systems of $(\mathbb{B}, \mathfrak{I})$ and $\left(\mathbb{S}^{\prime}, \mathfrak{T}^{\prime}\right)$ respectively, then there are a bijection $\beta: \nabla \simeq \nabla^{\prime}$ and a family $\left(q_{a}\right)_{a \in \nabla^{\circ}}$ of powers of $p^{*}$ with ${ }^{t} F(\beta(\alpha))=q_{a}$ a for all $\alpha \in \nabla$.

The famous uniqueness theorem of Chevalley [5, p. 53] tells us conversely that each special homomorphism $\psi: X^{\prime} \hookrightarrow X$ comes from an isogeny $F: B \rightarrow B^{\prime}$ such that $F(\mathfrak{I})=\mathfrak{I}^{\prime}$, which is determined uniquely up to inner automorphisms by the $k$-rational points of $\mathfrak{\Psi}$. On the other hand for each reduced root system $(X, \nabla)$ there exists a connected semisimple $k$-group scheme $(B, \widetilde{X})$, determined uniquely up to isomorphisms, having $(X, \nabla)$ as its root system (the existence theorem of Chevalley). The connected semisimple $k$ group scheme determined by the root system $(X, \nabla)$ is denoted by $\mathbb{B}(X, \nabla)$.

An isogeny of connected semisimple $k$-group schemes $f: B \rightarrow B^{\prime}$ (or the corresponding special homomorphism $\left.{ }^{t} \mathrm{~F}: X^{\prime} \hookrightarrow X\right)$ is called standard if all the indices $q_{a}$ are equal to 1 . The following facts concerning standard isogenies are well known. Recall that $\mathcal{S}(\Gamma)$ denotes the diagonalizable $k$-group scheme represented by a finitely generated Z-module $\Gamma$ (\$0.2).

2.1. Lemma. Let $\mathrm{F}: B \rightarrow B^{\prime}$ be a standard isogeny of connected semisimple k-group schemes, $\mathfrak{\Psi}$ a maximal torus of $\mathbb{P}, \mathfrak{I}^{\prime}=\mathfrak{F}(\mathfrak{T}), X=X(\mathfrak{T})$ and $X^{\prime}=X\left(\mathfrak{T}^{\prime}\right)$. Via the injection ${ }^{t} \mathrm{~F}: X^{\prime} \hookrightarrow X$, we view $X^{\prime}$ as a subgroup of $X$.

(1) $\operatorname{Rex}(F)=\operatorname{Rex}(F \mid \mathfrak{T})=\mathscr{S}\left(X / X^{\prime}\right)$.

(2) $\operatorname{Rex}(f) \subset \mathcal{C} \operatorname{ent}(\mathbb{F})$.

(3) $\operatorname{sent}(B)=S\left(X / X_{0}\right)$.

(4) $\operatorname{Rex}(\uparrow)$ is etale if and only if $\left(p^{*},\left[X: X^{\prime}\right]\right)=1$. Hence $(\circledast, \cap)$ is a p.etale group covering of $\beta^{\prime}$ ' in this case.

(5) If $(\mathfrak{F}, \gamma)$ is an etale group covering of $B^{\prime}$, then $\mathfrak{F}$ is smooth connected semisimple and $\gamma$ a standard isogeny.

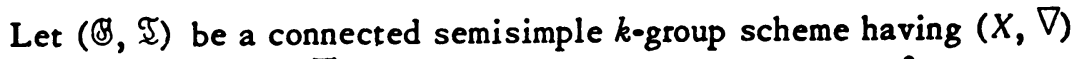
as its root system. Let $\bar{X} / X$ denote the largest subgroup of $X^{0} / X$ whose order is relatively prime to $p^{*}$. Hence $\left[X^{0}: \bar{X}\right]=$ a power of $p^{*}$.

2.2. Theorem. Let $k$ be algebraically closed. (i) The standard isogeny $\mathbb{B}(\bar{X}, \nabla) \rightarrow \mathbb{B}(X, \nabla)=\mathbb{B}$ induced from the inclusion of root systems $(X, \nabla)$ $\hookrightarrow(\bar{X}, \nabla)$ is a universal group covering, as well as a p-universal group covering, of $B$.

(ii) In particular the following conditions are equivalent to each other: (a) \& is (SC); (b) \& is (SC) ${ }_{p}$; (c) $X=\bar{X}$; (d) $\left[X^{0}: X\right]=$ a power of $p^{*}$.

Proof. This theorem follows driectly from Lemma 2.1, since every etale 
group covering of $B$ is (essentially) obtained as the standard isogeny $\mathbb{B}(Y, \nabla)$ $\rightarrow B(X, \nabla)$ induced from the inclusion $(X, \nabla) \hookrightarrow(Y, \nabla)$ for some subgroup $X$ $C Y \subset X^{0}$ with $\left(p^{*},[Y: X]\right)=1$. Q.E.D.

2.3. Corollary. If $k$ is algebraically closed, for a connected semisimple k-group scheme $B$, the following facts hold:

(1) The dual Hopf algebra hy(\$) ${ }^{0}$ is finitely generated.

(2) $\bar{B}=$ Spec $(\text { hy }(B))^{0}$ ) is smooth connected semisimple and (SC).

(3) The canonical epimorphism $\bar{B} \rightarrow B$ is a p-etale group covering.

(4) hy(B) $\simeq$ hy(B).

(5) hy(B) $0 / / O(\mathbb{B})$ is finite dimensional over $k$.

(6) $\bar{B}$ is a universal group covering, as well as a p-universal group covering, of $B$.

This follows directly from Lemma 1.8 and Theorem 2.2.

3. The case of semisimple group schemes. In this section we generalize the results of $\$ 2$ to the case of perfect ground field. Suppose $k$ is perfect throughout $\$ 3$. Let $\&$ be a connected smooth semisimple affine algebraic $k$ group scheme. This means that the $\bar{k}$-group scheme $\otimes \otimes \bar{k}$ is a Chevalley $\bar{k}$ group scheme, where $\bar{k}=$ the algebraic closure of $k$. But of course $\&$ itself is not necessarily of Chevalley type.

If $H$ is an arbitrary Hopf algebra over $k$, the dual $\bar{k}$-Hopf algebra $(H \otimes \bar{k})^{0}$ contains $H^{0} \otimes \bar{k}$ as a $\bar{k}$-sub-Hopf algebra. If $H$ is cocommutative and $(H \otimes \bar{k})^{0}$ is finitely generated over $\bar{k}$, then it follows from 0.1.1(a) that $H^{0}$ is finitely generated over $k$. In particular put $H=$ hy(B) the hyperalgebra of B. Then $H \otimes \bar{k}=\mathrm{hy}_{\bar{k}}(\mathbb{B} \otimes \bar{k})$ is the $\bar{k}$-hyperalgebra of the $\bar{k}$-group scheme $\otimes \nabla$. Since $(H \otimes \bar{k})^{0}$ is finitely generated over $\bar{k}$ by Corollary $2.3(1)$, it follows that $H^{0}$ is finitely generated over $k$. Let $\bar{B}=\operatorname{Spec}\left(H^{0}\right)$ and $(\otimes \otimes \bar{k})^{*}$ $=\operatorname{Spec}_{\bar{k}}\left((H \otimes \bar{k})^{0}\right)$. The canonical inclusions of $\bar{k} \cdot$ Hopf algebras

$$
O(\otimes) \otimes \bar{k} \hookrightarrow H^{0} \otimes \bar{k} \hookrightarrow(H \otimes \bar{k})^{0}
$$

induce epimorphisms of affine algebraic $\bar{k}$-group schemes

$$
B \otimes \bar{k} \leftarrow \bar{B} \otimes \bar{k} \leftarrow(\mathbb{B} \otimes \bar{k})^{*}
$$

whose composite is a universal, as well as a $p$-universal, group covering of $\left(8 \otimes \bar{k}\right.$ by Corollary 2.3(6). Applying the functor hy ${ }_{k}($ ?), one can easily conclude that the canonical epimorphism of affine algebraic $k$-group schemes $\bar{B} \rightarrow$ (B) (induced from $O(B) \hookrightarrow H^{0}$ ) is a p-etale group covering.

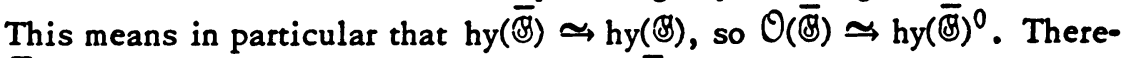
fore $\bar{B}$ is (SC) by Lemma 1.8. It follows that $\bar{B}$ is a universal, as well as a 
p-universal, group covering of $B$. Notice that $\bar{B}$ is connected smooth semisimple (since isogenous to $B$ ).

In particular if $B$ is $(S C)_{p}$, then $\bar{B} \simeq \mathbb{B}$, so $B$ is $(S C)$. Summarizing we have

3.1. Theorem. Suppose $k$ is perfect and let $\&$ be a connected smooth semisimple affine algebraic k-group scheme. Then the following facts hold.

(1) The dual Hopf algebra hy(B) ${ }^{0}$ is finitely generated.

(2) $\bar{B}=\operatorname{Spec}\left(\mathrm{hy}(\mathbb{B})^{0}\right)$ is smooth connected semisimple and (SC).

(3) The canonical epimorphism $\bar{B} \rightarrow \mathbb{B}$ is a p-etale group covering.

(4) hy(B) $\simeq$ hy(B).

(5) hy(B) $\% / \cap(\&)$ is finite dimensional over $k$.

(6) $\bar{B}$ is a universal, as well as a p-universal, group covering of 8 .

(7) B is $(S C)_{p}$ if and only if $(S C)$.

The purpose of the rest of this section is to prove that the canonical epimorphism $(\& \otimes \bar{k})^{*} \rightarrow \bar{B} \otimes \bar{k}$ is isomorphic.

In general if $\overline{\mathscr{F}}$ is a $\bar{k}$-group scheme, we mean by॰a $k$-form of $\overline{\mathscr{T}}$, a $k$ group scheme $\mathcal{F}_{2}$ with $\tilde{F} \otimes \bar{k} \simeq \overline{F_{2}}$. If $\bar{F}: \overline{\mathscr{F}} \rightarrow \otimes \otimes \bar{k}$ is a homomorphism of $\bar{k}$-group schemes, we say that $\bar{F}$ is defined over $k$ if there are a $k$-form $\mathscr{F}$ of $\overline{\mathscr{F}}$ and a homomorphism of $k$-group schemes $\mathrm{f:} \mathscr{F}_{2} \rightarrow$ with

$$
\bar{F}: \overline{\mathscr{F}} \simeq \mathscr{S} \otimes \bar{k} \stackrel{f \otimes \vec{k}}{\longrightarrow} \otimes \otimes \bar{k} .
$$

3.2. Lemma. The canonical epimorphism of $\bar{k} \cdot$ group schemes, $(\otimes \otimes \bar{k})^{*}$ $\rightarrow \otimes \nabla$, is defined over some finite extension field $l$ of $k$.

Proof. Let $k_{0}$ be the prime field in $k$. Each Chevalley $\bar{k}$-group scheme $\notin(X, \nabla)$ has a canonical $k_{0}$-form $[5$, p. 53] and the standard isogeny $\&(\bar{X}, \nabla)$ $\rightarrow \mathbb{B}(X, \nabla)$ induced from the inclusion $X \hookrightarrow \bar{X}$ can be taken to be defined over $k_{0}[5, \mathrm{p} .60]$. Now that $B$ is semisimple, there is a unique root system $(X, \nabla)$ with $\& \otimes \bar{k} \simeq \mathbb{B}(X, \nabla)$. By the universal mapping property of universal group coverings, we have a commutative diagram

$$
\begin{aligned}
& (\otimes \otimes)^{*} \simeq \mathbb{B}(\bar{X}, \nabla) \\
& \text { cano } \downarrow \downarrow \text { cano } \\
& \otimes \otimes \bar{k} \simeq \mathbb{E}(X, \nabla)
\end{aligned}
$$

where the right vertical arrow is defined over $k_{0}$. Since $\&$ and $\&(X, \nabla)$ are both algebraic, there is a finite extension $l / k$ such that the isomorphism (B) $\otimes \bar{k} \simeq \mathbb{B}(X, \nabla)$ is defined over $l$. The lemma follows from this directly. Q.E.D. 
If we put $H=$ hy(B) as before, the above lemma implies that there is an l-sub-Hopf algebra $A$ of $(H \otimes \bar{k})^{0}$ with

$$
\Theta(\otimes) \otimes_{k} l \subset A \text { and } A \otimes_{l} \bar{k}=(H \otimes \bar{k})^{0} \text {. }
$$

If we put $\Omega=\operatorname{Spec}_{l}(A)$, then the canonical epimorphism of l-group schemes $\mathfrak{R} \rightarrow B \otimes_{k} l$ is an etale group covering, since so is

$$
\left(B \otimes_{k} \bar{k}\right)^{*}=2 \otimes_{l} \bar{k} \rightarrow\left(B \otimes_{k} l\right) \otimes_{l} \bar{k}=B \otimes_{k} \bar{k} .
$$

Hence hy ${ }_{l}(\Omega) \simeq H \otimes_{k} l$, so hy ${ }_{l}(\Omega)^{0} \simeq H^{0} \otimes_{k} l$. (In general, if $l / k$ is finite, $\left(B \otimes_{k} l\right)^{0}=B^{0} \otimes_{k} l$ for all $k$-algebras B.) Thus we get a commutative diagram:

$$
\begin{aligned}
& \operatorname{hy}_{l}(\Omega)^{0} \simeq H^{0} \otimes_{k} l \\
& \cup U
\end{aligned}
$$

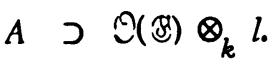

Applying the functor ? $\otimes_{l} \bar{k}$, we obtain

$$
\begin{aligned}
\operatorname{hy}_{l}(\Omega)^{0} \otimes_{l} \bar{k} & \simeq H^{0} \otimes_{k} \bar{k} \\
\cup & \cup \\
A \otimes_{l} \bar{k} & \supset \Theta\left((\mathbb{S}) \otimes_{k} \bar{k} .\right.
\end{aligned}
$$

But since $A \otimes_{l} \bar{k}=\left(H \otimes_{k} \bar{k}\right)^{0}$, this means that $\left(H \otimes_{k} \bar{k}\right)^{0}=H^{0} \otimes_{k} \bar{k}$ and hence that $(\otimes \otimes \bar{k})^{*} \simeq \mathbb{\otimes} \otimes \bar{k}$. Thus we proved

3.3. Theorem. Suppose $k$ is perfect. Let $\&$ be a connected smooth semisimple affine algebraic k-group scheme. If $\overline{\mathbb{B}}$ is the universal group covering of $B$, then $\bar{B} \otimes \bar{k}$ is the universal group covering of the $\bar{k}$-scheme $B \otimes \bar{k}$. In particular $\&$ is (SC) (or equivalently $(S C)_{p}$ ) if and only if $\& \otimes \bar{k}$ is (SC) (or equivalently $(S)_{p}$ ) as a $\bar{k}$-group scheme.

4. Semisimple hyperalgebras. In this section assume $k$ is perfect. We prove that every semisimple hyperalgebra of finite type is the hyperalgebra of some connected semisimple $k$-group scheme. Since connected semisimple $k$-group schemes have the universal group covering, it will follow from Theorem 3.1 that the category of (SC) semisimple $k$-group schemes is equivalent to the category of semisimple hyperalgebras of finite type.

A hyperalgebra of finite type $J$ is called representable if the dual Hopf algebra $J^{0}$ is dense in $J^{*}$, or equivalently if there is an affine algebraic $k$ group scheme $\&$ with $J \subset$ hy(\&). Here the $k$-group scheme $\&$ can be taken so that $J$ is dense in hy(B) (i.e., $O(\mathbb{B}) \subset J^{0}$ ) and hence in particular that $\&$ is connected. Each finite type hyperalgebra $J$ contains the smallest normal subhyperalgebra $K$ such that the quotient hyperalgebra $J / / K$ is representable; 
$K$ is the Hopf kernel of $J \rightarrow J^{00}$. We shall denote $K=K_{\text {rep }}(J)$. It follows from Dieudonné [ 1, Proposition 20, p. 365] that $K_{\text {sep }}(J)$ is contained in the center of $J$.

4.1. Lemma. Let $J$ be a smooth byperalgebra of finite type and $K$ a finite normal subhyperalgebra of $\mathrm{J}$. If $\mathrm{J} / / \mathrm{K}$ is representable, then so is $\mathrm{J}$.

Proof. We can assume $p>0$. With a sufficiently large positive integer $r$, let $V^{r}: J \rightarrow J^{\left(p^{r}\right)}$ denote the r-times iterated Verschiebung map of $J(\$ 0.1)$. Then $V^{r}$ is surjective ( $(0.3)$ with a finite Hopf kernel containing $K$ and hence it induces a surjective homomorphism $J / / K \rightarrow J^{\left(p^{r}\right)}$ which has also a finite Hopf kernel. If, in general, $l \mid k$ is a field extension and if $L$ is a representable hyperalgebra over $k$, then the l-hyperalgebra $l \otimes L$ is representable too. Since now $k$ is perfect, we have $J=\left(J^{\left(p^{r}\right)}\right)^{\left(p^{-r}\right)}$. Hence it suffices to show the representability of $J^{\left(p^{r}\right)}$, which will follow from the next lemma. Q.E.D.

4.2. Lemma. If $J$ is a representable hyperalgebra of finite type and $K$ a finite normal subbyperalgebra of $J$, then the quotient $J / / K$ also is repre. sentable.

Proof. Imbed $J$ into hy(B) as a dense subhyperalgebra for some connected affine algebraic $k$-group scheme $\mathbb{B}$. Since $[J, K] \subset K$, it follows from $0.3 .4(e)$ that [hy( $(\mathbb{B}), K] \subset K$. Hence $K$ is a normal subhyperalgebra of hy(B). Since any finite dimensional subhyperalgebra of hy(B) is closed (0.3.4(a)), it follows that there is a unique closed normal connected subgroup scheme $\mathcal{F}$ of $\&$ with $K=h y(\mathcal{F})$. The induced inclusion

$$
J / / K \hookrightarrow h y(\mathbb{B}) / / \operatorname{hy}(\mathfrak{L})=h y(\mathbb{B} / / 2)
$$

proves the representability of $J / / K$. Q.E.D.

4.3. Proposition. Let J be a smooth byperalgebra of finite type. Then $K_{\text {rep }}(J)$ is smooth too.

Proof. Let $K_{\mathrm{sm}}$ denote the smooth part of $K=K_{\text {rep }}(J)(\$ 0.3)$. Since $K$ is central, we can consider the quotient hyperalgebra $J / / K_{s m}$ which has a finite dimensional normal subhyperalgebra $K / / K_{\mathrm{sm}}(\$ 0.3)$. Since the quotient $\left(J / / K_{s \mathrm{~m}}\right) / /\left(K / / K_{s \mathrm{~m}}\right)=J / / K$ is representable, it follows from Lemma 4.1 that so is $J / / K_{s \mathrm{~m}}$. By the definition of $K_{\mathrm{rep}}(J)$, this proves that $K=K_{\mathrm{sm}}$. Q.E.D.

For each hyperalgebra $J$, we have defined the derived subhyperalgebra $[J, J]$ in $\$ 0.1$. Hence we can define the derived series $\left\{J^{(\nu)}\right\}$ of $J$ by $J^{(\nu)}=$ $=\left[J^{(\nu-1)}, J^{(\nu-1)}\right]$ and $J^{(0)}=J$. The hyperalgebra $J$ is called solvable if $J^{(N)}=k$ for some $N$.

Let $J$ be a smooth hyperalgebra of finite type. The set of normal smooth 
solvable subhyperalgebras of $J$ contains clearly the largest element denoted by $\operatorname{rad}(J)$, which we shall call the radical of $J . J$ is called semisimple if $\operatorname{rad}(J)=k$. In general $J / / \operatorname{rad}(J)$ is semisimple for any smooth hyperalgebra $J$ of finite type.

4.4. Proposition. Semisimple smooth byperalgebras of finite type are representable.

Proof. If $J$ is such a hyperalgebra, then $K_{\text {rep }}(J)$ is smooth and central by Proposition 4.3 and hence is trivial. Q.E.D.

Let $J$ be a smooth semisimple hyperalgebra of finite type. Embed as usual $J$ into the hyperalgebra hy(B) as a dense subhyperalgebra for some connected affine algebriac smooth $k$-group scheme $\mathbb{B}$. Let $\mathrm{xab}(\mathbb{B})$ denote the radical of $\mathbb{B}$.

4.5. Lemma. The hyperalgebra $J \cap h y(\operatorname{rab}(\mathbb{B}))=K$ is finite deminsional.

Proof. $K$ is clearly normal solvable in $J$. Since $[J, K] \subset K$, it follows from $J=J_{s \mathrm{~m}}$ that $\left[J, K_{s \mathrm{~m}}\right] \subset K_{s \mathrm{~m}}$, where ( $)_{s \mathrm{~m}}$ denotes the smooth part of the hyperalgebra. Thus $K_{s \mathrm{~m}}$ is normal smooth solvable in $J$ and is trivial by assumption. This means that $K$ is finite dimensional. Q.E.D.

Notice that $J / / K \subset$ hy( $(\mathbb{S a b}(\mathbb{B}))$ is also dense. Hence it follows from $0.3 .4(f)$ that

$$
[J / / K, J / / K]=[\mathrm{hy}(\& / \mathrm{rab}(\&)), \mathrm{hy}(\& / \mathrm{rab}(\&))) \text {. }
$$

Since $B / \mathrm{rab}(B)$ is a semisimple $k$-group scheme, it coincides with the derived group. This means that

$$
[J / / K, J / / K)=\operatorname{hy}(\& / \operatorname{rab}(\$))
$$

and hence, in particular, that $J / / K=[J / / K, J / / K]$, or equivalently that $J=$ $[j, J] K$. Then the quotient hyperalgebra $J / \Lambda J, J]$ is finite and smooth and therefore $J=[J, J]$. This proves that $J$ is a closed subhyperalgebra of hy( (8) $(0.3 .4(g))$. Since $J$ is dense in hy(\$), it follows that $J=$ hy( $(\mathbb{S})$. Now hy( $\mathrm{xab}(\mathbb{B}))$ is a normal smooth solvable subhyperalgebra of $J$. Since $J$ is semisimple, it follows that $\mathrm{hy}(\operatorname{xa\delta }(\mathbb{B}))=k$ and hence that $\operatorname{xa}(\mathbb{B})=(e)$ or that $\&$ is semisimple. Thus we have proved

4.6. Theorem. If $k$ is perfect each smooth semisimple hyperalgebra of finite type is the hyperalgebra of some connected semisimple k-group scheme.

Conversely we have

4.7. Proposition. Let \& be a connected smooth affine algebriac k-group

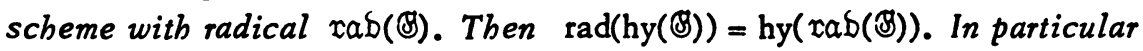


the k-group scheme $\mathbb{B}$ is semisimple if and only if the byperalgebra hy(\&) is.

Proof. The inclusion hy( $\mathrm{xab}(\mathbb{B})) \subset \operatorname{rad}(\mathrm{hy}(\mathbb{B}))$ is clear. Let $K$ be a normal smooth solvable subhyperalgebra of hy(\&) and $A(K)$ the algebraic hull of $K$ in hy(B) (\$0.3). Since $K$ is contained in the smooth part $A(K)_{\mathrm{sm}}$, which is also closed, it follows that $A(K)$ is smooth. Since $[A(K), A(K)]=[K, K]$, the solvability of $A(K)$ follows. Similarly the equality [hy $(\&), A(K)]=[$ hy( $(\&)$, $K]$ (0.3.4(d)) implies that $A(K)$ is normal in hy(B). Let $\mathcal{F}_{2}$ be a unique closed connected normal subgroup scheme of $\&$ with hy $\left(F_{2}\right)=A(K)$. Then $\mathscr{F}_{2}$ is solvable smooth and hence contained in $\mathrm{xab}(\mathbb{B})$. Therefore $K \subset$ hy( $\mathrm{xab}(\mathbb{B})$ ). Q.E.D.

By virtue of Theorem 3.1, Theorem 4.6 has the following corollary:

4.8. Theorem. Suppose $k$ is perfect. If $J$ is a smooth semisimple byperalgebra of finite type, then the dual Hopf algebra $J^{0}$ is finitely generated and the corresponidng affine k-group scheme $\operatorname{Spec}\left(J^{0}\right)$ is (SC) semisimple and has $J$ as its byperalgebra. The functors $J \mapsto \operatorname{Spec}\left(J^{0}\right)$ and $\& \mapsto$ hy(\&) give rise to an equivalence between the categories of smooth semisimple byperalgebras of finite type and of (SC) semisimple k-group schemes.

5. An example of nonreductive (SC) $k$-group schemes. To conclude this paper we shall provide a simple example of (SC) affine algebraic $k$-group schemes which are not reductive.

In this section we shall assume $k=\bar{k}$ with $p>0$. For each left finitely generated $k[F]$-module $M$, let $\mathfrak{U}(M)=\operatorname{Spec}\left(U^{[p]}(M)\right)$ denote the corresponding commutative unipotent affine algebraic $k$-group scheme killed by the Verschiebung map (see $\$ 0.2$ ). Let $\bar{B}$ denote an arbitrary affine algebraic $k$-group scheme.

5.1. Definition. Let $M$ be a finitely generated left $k[F]$-module. View the affine ring $\Theta(\bar{B})$ as a left $k[F]$-module via $F a=a^{p}, a \in \Theta(\bar{F})$. A right $\theta$ (ब) -comodule structure on $M, \rho: M \rightarrow M \otimes O(\bar{B})$, which is $k[F]$-linear, is said to be compatible with the $k[F]$-module structure. A left $k[F]-\bar{B}$-module means a finitely generated left $k[F]$-module with a right $O(\bar{E})$-comodule structure compatible with the $k[F]$-module structure.

5.2. Lemma. The k-group scheme actions as automorphisms of $k$-group schemes $\mathfrak{U}(M) \times \bar{B} \rightarrow \mathfrak{U}(M)$ correspond bijectively with the right $\Theta(\bar{B})-c 0$ module structures on $M$ which are compatible with the $k[F]$-module structure.

Proof. The $k$-group scheme actions of the above mentioned type can be identified with those right comodule structures 


$$
\rho: U^{[p]}(M) \rightarrow U^{[p]}(M) \otimes \Theta(\overline{\mathbb{B}})
$$

which are compatible with the Hopf algebra structure on $U^{[p]}(M)$. This means in particular that $\rho(M) \subset M \otimes O(\bar{F})$ and that the restricted coaction $\rho \mid M$ is compatible with the $k[F]$-module structure. Conversely if $M$ is a left $k[F]$ ब․module with coaction $\sigma: M \rightarrow M \otimes \Theta(\bar{B})$ then the extended algebra map $\sigma:$ $U^{[p]}(M) \rightarrow U^{[p]}(M) \otimes O(\bar{F})$ is easily seen to be compatible with the Hopf algebra structure of $U^{[p]}(M)$. Q.E.D.

In the following let $M$ be a (finitely generated) left $k[F]-\overline{8}-$ module such that $\mathfrak{U}(M)$ is connected (see $\$ 0.2$ ). The isomorphism classes of exact sequences of homomorphisms of affine $k$-group schemes, $1 \rightarrow \mathfrak{U}(M) \rightarrow B \rightarrow \bar{B}$ $\rightarrow 1$, the right action of $\bar{B}$ on $\mathfrak{U}(M)$ induced from which coincides with the original action, form an abelian group in a usual manner, which we shall write as $\operatorname{Ext}(\overline{\mathbb{F}}, \mathfrak{U}(M))$. The neutral element is supplied by the semidirect product $\bar{B} \times{ }_{s} \mathfrak{U}(M)$.

5.3. Definition. The $k[F]-\bar{B}-$ module $M$ is of type (\#) if the following condition is satisfied:

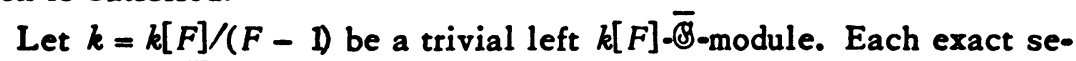
quence of $k[F] \cdot \bar{B}-$ modules of the form $0 \rightarrow M \rightarrow N \rightarrow k \rightarrow 0$ splits as $k[F]$ modules.

5.4. Proposition. If $\bar{B}$ is (SC) and the k[.F]-可-module $M$ is of type (\#), then each k-group scheme $\mathbb{B}$ in $\operatorname{Ext}(\bar{B}, \mathfrak{U}(M)$ ) is (SC) too.

Proof. Let $B \in \operatorname{Ext}(\bar{B}, \mathfrak{U}(M)),(\mathscr{F}, \gamma)$ an etale group covering of $\mathbb{B}$, and $\mathfrak{F}_{u}$ the connected component of $\gamma^{-1}(M(M))$. We have a commutative diagram:

$$
\begin{aligned}
& 1 \rightarrow \mathfrak{F}_{u} \rightarrow \mathscr{F}_{2} \rightarrow \mathscr{F}_{2} / F_{2} \rightarrow 1 \\
& \text { a\} \quad \gamma \downarrow \beta \downarrow } \\
{1 \rightarrow \mathfrak{u}(M) \rightarrow \mathbb{B} \rightarrow \infty}
\end{aligned}
$$

where the homomorphisms $\alpha, \beta$ and $\gamma$ are all etale group coverings. Since $\bar{B}$ is (SC), $\beta$ is an isomorphism, via which $\bar{B}$ operates on $\mathscr{L}_{u}$ which is commutative unipotent and killed by the Verschiebung, because hy $(a)$ : hy $\left(\xi_{u}\right)$ $\simeq$ hy $(\mathfrak{U}(M))$. Hence we can write $\mathfrak{L}_{u}=\mathfrak{U}(N)$ for some left $k[F]$ - $\bar{B}$-module $N$. The homomorphism $a$ induces an injection of $k[F]-\bar{B}-$-modules $M \hookrightarrow N$ and the quotient module $N / M$, which represents the kernel of $a$, which is etale, is therefore a direct sum of some finite number of copies of the trivial $k[F]$. $\bar{B}$-module $k=k[F] /(F-1)$. Say $N / M \simeq k^{r}$. The exact sequence of $k[F]$. $\bar{B}$. modules $0 \rightarrow M \rightarrow N \rightarrow k^{r} \rightarrow 0$ splits as $k[F]$-modules, because $M$ is of type (\#). But since $\mathfrak{U}(\dot{N})$ is connected, this means that $r=0$ and hence that $a$ is an isomorphism. Hence $\gamma$ is an isomorphism and 8 is (SC). Q.E.D. 
Suppose that the affine algebraic k-group scheme $\bar{B}$ is connected smooth and put $A=\Theta(\bar{B})$. Let $V$ be a finite dimensional right $A$-comodule with the structure map $\rho: V \rightarrow V \otimes A$ and put $M=k[F] \otimes V$. Extending $\rho k[F]$ linearly, we make $M$ into a $k[F]$. $\bar{S}$-module.

5.5. Proposition. Suppose that $\bar{B}$ is connected smooth. Then the $k[F]$ 可-module $M=k[F] \otimes V$ is of type (\#) if and only if $V^{\bar{S}}=0$.

Proof. The 'only if' part is easy. Suppose that $V^{\mathbb{C}}=0$. Let $V \otimes_{i} A$ be the tensor product of $V$ and $A$ over $k$ with the defining relation

$$
\lambda v \otimes_{i} a=v \otimes_{i} \lambda^{p^{i}} a \text { for } \lambda \epsilon k, v \in V, a \in A \text {. }
$$

The space $M \otimes A$ is the direct sum of $k F^{i} \otimes\left(V \otimes_{i} A\right), i \geq 0$. Since $A$ is smooth, the $k$-linear maps

$$
V \otimes_{i} A \hookrightarrow V \otimes_{i+j} A, \quad v \otimes_{i} a \mapsto\left(v \otimes_{i} a\right)^{(j)} \underset{\operatorname{def}}{=} v \otimes_{j+j} a^{p^{j}}
$$

are injective. Let $0 \rightarrow M \rightarrow N \rightarrow k[F] /(F-1) \rightarrow 0$ be an exact sequence of $k[F]$ - $\bar{B}$-modules. Thus we can write $N=M \oplus k e$ with $F e-e=\Sigma_{i} F^{i} \otimes v_{i}$ $\left(v_{i} \in V\right)$ and $\rho(e)-e \otimes 1=\Sigma_{i} F^{i} \otimes x_{i}\left(x_{i} \in V \otimes_{i} A\right)$. Since $\rho$ is $k[F]$-linear, it follows that $x_{i}=\partial\left(v_{i}\right)^{(i)}+x_{i-1}^{(1)}$ for $i>0$ and $x_{0}=\partial\left(v_{0}\right)$, where $\partial(v)=$ $v \otimes 1-\rho(v) \in V \otimes A\left(=V \otimes_{0} A\right)$ for $v \in V$. Hence if we put $u_{i}=v_{i}+\cdots+v_{0}$ $\epsilon V$, then $x_{i}=\partial\left(u_{i}\right)^{(i)}$. Since the $k$-linear map $\partial: V \rightarrow V \otimes A$ is injective by assumption, it follows that $u_{i}$ are equal to zero for almost all $i$. Hence we can well define an element of $M m=-\Sigma F^{i} \otimes u_{i}$. It is easy to see $F m-$ $m=F e-e$. This means that the $k[F]-\bar{B}-$ module $M$ is of type (\#). Q.E.D.

If we take $\bar{B}$ to be an (SC) semisimple $k$-group scheme and $V$ a nontrivial irreducible $k$-ब্ত্-module, then $V^{\bar{\Phi}}=0$ clearly and hence each element of $\operatorname{Ext}\left(\bar{B}, \mathscr{P}_{a}(V)\right)$ provides an example of nonreductive (SC) $k$-group schemes, where $\mathscr{P}_{a}(V)=\operatorname{Spec}(S(V))$ represented by the symmetric algebra $S(V)$ on $V$, which is a Hopf algebra having $V$ as primitive elements.

Added in proof. All subhyperalgebras of a finite type hyperalgebra is of finite type by definition. Each quotient hyperalgebra, which must be of the form $J / / K$ with $K$ a normal subhyperalgebra by $(0.1 .2)$, is also of finite type, when $J$ is $\left[\mathrm{T}_{\mathrm{II}}, 5.5 .2 .1\right]$. This is implicitly used in $\$ 4$.

\section{REFERENCES}

[DG] M. Demazure and P. Gabriel, Groupes algébriques. Tome I: Géométrie algébrique, généralités, groupes commutatifs, North-Holland, Amsterdam, 1970.

$\left[\mathrm{T}_{\mathrm{I}}\right]$ M. Takeuchi, Tangent coalgebras and hyperalgebras. I, Japan. J. Math. 42 (1974), 1-43.

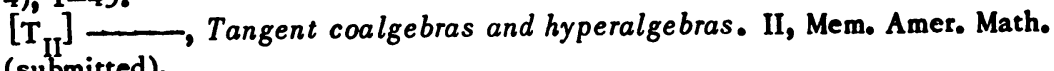
Soc (submitted).

1. J. Dieudonné, Lie groups and Lie hyperalgebras over a field of characteristic $p>0$. VI, Amer. J. Math. 79 (1957), 331-388. MR 20 \#931. 
2. G. Hochschild, Algebraic groups and Hopf algebras, Illinois J. Math. 14 (1970), 52-65. MR 41 \#1742.

3. N. Iwahori, The theory of Lie algebras and Chevalley groups, Seminar Notes 12, 13, University of Tokyo, Tokyo, 1965. (Japanese)

4. M. Miyanishi, Une caractérisation d'un groupe algébrique simplement connexe, Illinois J. Math. 16 (1972), 639-650. MR 46 \#9052.

5. I. Satake, Classification theory of semi-simple algebraic groups, Lecture Notes in Pure and Appl. Math., 3, Dekker, New York, 1971. MR 47 \#135.

6. M. E. Sweedler, Hopf algebras, Math. Lecture Note Series, Benjamin, New York, 1969. MR 40 \#5705.

7. M. Takeuchi, A correspondence between Hopf ideals and sub-Hopf algebras, Manuscripta Math. 7 (1972), 251-270. MR 48 \#328.

DEPARTMENT OF THE FOUNDATIONS OF MATHEMATICAL SCIENCES, TOKYO UNIVERSITY OF EDUCATION, OTSUKA, BUNKYO-KU, TOKYO, JAPAN

Current address: Department of Mathematics, University of Tsukuba, Ibaraki, 300-31 Japan 